\title{
TWO-DIMENSIONAL AXISYMMETRIC COLLAPSE OF THERMALLY UNSTABLE PRIMORDIAL CLOUDS
}

\author{
Chang Hyun Baek and Hyesung Kang \\ Department of Earth Sciences, Pusan National University, Pusan 609-735, Korea; \\ chbaek@comet.es.pusan.ac.kr, kang@uju.es.pusan.ac.kr \\ AND \\ DONGSU RYu \\ Department of Astronomy and Space Science, Chungnam National University, 220 Kung-dong, \\ Yusong-ku, Daejeon 305-764, Korea; ryu@canopus.chungnam.ac.kr \\ Received 2002 August 8; accepted 2002 October 29
}

\begin{abstract}
We have performed two-dimensional hydrodynamic simulations of the collapse of isolated axisymmetric clouds condensing via radiative cooling in a primordial background gas. In order to study the development of the so-called shape instability, we have considered two types of axisymmetric clouds, oblate and prolate clouds of various sizes and with axial ratios of $0.5 \leq R_{c, R} / R_{c, z} \leq 2$. We find that the degree of oblateness or prolateness is enhanced during the initial cooling phase. However, it can be reversed later, if the initial contrast in cooling times between the cloud gas and the background gas is much greater than 1 . In such cases an oblate cloud collapses to a structure composed of an outer thin disk and a central prolate component. A prolate cloud, on the other hand, becomes a thin cigar-shaped structure with a central dense oblate component. The reversal of shape in the central part of the cooled clouds is due to supersonic motions either along the disk plane in the case of oblate clouds or along the symmetry axis in the case of prolate clouds. For a background gas of $T_{h}=1.7 \times 10^{6} \mathrm{~K}$ and $n_{h}=0.1 \mathrm{~cm}^{-3}$ in a protogalactic halo environment, the mean density of the cloud gas that has cooled to $10^{4} \mathrm{~K}$ increases to $100 n_{h}$ or so in our simulations where nonequilibrium cooling is adopted and the background gas cools too. The spherical Jeans mass of such gas is estimated to be about $M_{\mathrm{J}} \sim 5 \times 10^{7} M_{\odot}$. In order for cloud mass to exceed the Jeans mass and at the same time in order for the thermal instability to operate, the initial cloud size should be around $1 l_{\text {cool }}-1.5 l_{\text {cool }}$, where $l_{\text {cool }}$ is the cooling length.

Subject headings: globular clusters: general — hydrodynamics — instabilities
\end{abstract}

\section{INTRODUCTION}

In many areas of astrophysics, the thermal instability is often invoked to explain the condensation of cold dense clouds out of a hot background medium (e.g., Field 1965; Goldsmith 1970; Defouw 1970; Schwarz, McCray, \& Stein 1972; Fall \& Rees 1985; Balbus \& Soker 1989; VázquezSemadeni, Gazol, \& Scalo 2000; Koyama \& Inutsuka 2002; Kritsuk \& Norman 2002). In the simplistic picture of the thermal instability, the overdense region surrounded by the hotter background undergoes a "quasi-static compression" in near pressure equilibrium. This " near-equilibrium" case of the evolution, however, is valid only when the cloud is small enough to adjust to pressure change faster than it cools: its size must be much smaller than the distance that a sound wave travels in a cooling time, $l_{\text {cool }}$. The collapse of thermally unstable clouds in either X-ray cluster cooling flows or protogalactic halos has been studied previously by numerical simulations (e.g., David, Bregman, \& Seab 1988; Brinkman, Massaglia, \& Müller 1990; Kang, Lake, \& Ryu 2000 and references therein). These simulations showed that a spherically symmetric cloud cools and undergoes a supersonic compression, if the cloud size is $R_{c} \sim l_{\text {cool }}$. Such supersonic compression leads to a central density increase 2-3 orders of magnitude higher than what is expected from the isobaric compression. On the other hand, a small cloud of $R_{c}<l_{\text {cool }}$ cools isobarically and undergoes a quasi-static compression, while a big cloud of $R_{c}>l_{\text {cool cools nearly }}$ isochorically with only small density increase.
While most previous studies on the thermal instability considered the collapse of one-dimensional spherically symmetric clouds, Brinkman et al. (1990) simulated the collapse of a nonspherical, elongated blob in the two-dimensional polar geometry and showed the development of a "shape instability." As the perturbation is compressed by the background pressure, the compression wave travels the same distance with the sound speed (i.e., $l \sim c_{s} t$ ) along both the major and minor axes, and the induced infall velocity field is not radial. This causes the compressed region to be more elongated and enhances the degree of nonsphericity. They showed that an oblate cloud with an initial axial ratio of $b / a=0.447$ collapses to a flat pancake and argued that the evolution of oblate clouds becomes similar to that of onedimensional plane-parallel collapses. From this simulation one can deduce that the shape instability would also occur in a prolate cloud, resulting in a thin rod-shaped condensation. In Kang et al. (2000) we studied the effects of different geometries by one-dimensional plane-parallel and onedimensional spherical symmetric simulations of thermally unstable clouds using a piecewise parabolic method (PPM) hydrodynamic code with self-gravity and radiative cooling. We found that isotropic compression leads to much higher central density, accompanied by accelerated radiative cooling, in the spherically symmetric case, while the density increases only to the isobaric ratio of $\rho_{c} / \rho_{h}=\left(T_{h} \mu_{c} / T_{c} \mu_{h}\right)$ in the plane-parallel case. Here $\mu$ is the mean molecular weight and the subscripts $c$ and $h$ stand for cool and hot, respectively. Thus, we expect that the cloud shape is an 
important factor in the collapse and evolution of thermally unstable clouds. In this contribution, we explore in detail how nonspherical perturbations in a primordial environment evolve under the influence of the thermal instability.

In many models of globular cluster (GC) formation, dense protoglobular cluster clouds (PGCCs) are supposed to exist in pressure equilibrium with the hot gas in protogalaxies (e.g., Gunn 1980; Brown, Burkert, \& Truran 1991; Kumai, Basu, \& Fujimoto 1993). The model by Fall \& Rees (1985), which has been most widely adopted to explain the existence of such PGCCs, relies on the thermal instability for the formation of PGCCs in a protogalactic halo. In our study the parameters have been adjusted so the simulations for the nonlinear development of the thermal instability are applicable to the condensation of PGCCs, although the generic results should hold to the thermal instability of any object. We have ignored self-gravity because the gravitational timescale is much longer than the cooling timescale during the early collapse stage of PGCCs. However, the self-gravity should be important in the gravitational fragmentation of PGCCs during the later evolutionary stage when the clouds have cooled down to $10^{4} \mathrm{~K}$.

In $\S 2$ we describe our model and numerical method. The simulation results are presented in $\S 3$, followed by the conclusions in $\S 4$.

\section{HYDRODYNAMIC SIMULATIONS}

\subsection{Isolated Clouds in a Uniform Background Halo}

We expect that inside a protogalactic halo, density perturbations on a wide range of length scales exist and flow motions are likely turbulent. While the thermal instability under such realistic global pictures can be explored later (e.g., Vázquez-Semadeni et al. 2000; Kritsuk \& Norman 2002), here we first take a much simpler and local approach. We consider isolated overdense clouds embedded in a hot, uniform, and static background gas of $T_{h}=1.7 \times 10^{6} \mathrm{~K}$ and $n_{h}=0.1 \mathrm{~cm}^{-3}$. This temperature corresponds to that of an isothermal sphere with circular velocity $V_{c}=220 \mathrm{~km}$ $\mathrm{s}^{-1}$, representing the halo of disk galaxies like the Milky Way. Here $n_{h}$ is the background density of hydrogen nuclei. The value of $n_{h}=0.1 \mathrm{~cm}^{-3}$ is chosen as a fiducial value because then spheres of radius $R_{c} \sim l_{\text {cool }}$ would have mass scales relevant for $\mathrm{GC}$ formation (see $\S 2.3$ ). We assume that the ratio of $\mathrm{He} / \mathrm{H}$ number densities is $1 / 10$, so that the gas mass density is given by $\rho_{h}=\left(2.34 \times 10^{-24} \mathrm{~g}\right) n_{h}$.

The initial density of the overdense clouds, $n_{\text {cloud }}$, is assumed to decrease gradually from the center to edge,

$$
n_{\text {cloud }}=n_{h}\left(1+\delta \cos \left\{\frac{\pi}{2}\left[\left(\frac{R}{R_{c, R}}\right)^{2}+\left(\frac{z}{R_{c, z}}\right)^{2}\right]^{1 / 2}\right\}\right)
$$

for $\left(R / R_{c, R}\right)^{2}+\left(z / R_{c, z}\right)^{2} \leq 1$, where $R_{c, R}$ and $R_{c, z}$ are the cloud radii along the $R$ - and $z$-axes, respectively. The ratio of $R_{c, R} / R_{c, z}$ determines the shape of the initial clouds (see $\S 2.5$ ). The initial temperature throughout the clouds is set by the isobaric condition, i.e., $T_{\text {cloud }}=T_{h}\left(n_{h} / n_{\text {cloud }}\right)$. The amplitude of initial density perturbations, $\delta$, is a free parameter that determines the density contrast between the cloud center and the background. If this amplitude is linear (i.e., $\delta \ll 1)$ and if there are no heat sources that balance the radiative cooling of the background gas, then both the cloud and the background gas cool together and the thermal instability would not have enough time to grow. Because the cooling time scales as $t_{\text {cool }} \propto(1+\delta)^{-2}$, the contrast in $t_{\text {cool }}$ between the cloud and the background is small for small $\delta$. In real protogalaxies, however, the halo gas would be heated by possible energy sources such as supernova explosions, shocks, etc. If the background gas maintains a high temperature as a result of those energy inputs, perturbations would grow approximately under the isobaric condition until they become nonlinear. In addition, these heating processes likely induce turbulent flow motions and nonlinear density fluctuations in the halo medium as well. In numerical simulations the overall evolution proceeds faster for larger values of $\delta$ during the linear phase, but it becomes almost independent of the initial values of $\delta$, once perturbations become nonlinear. Since we do not include any background heating processes in our simulations, we need to have a reasonably large density contrast in order to see the nonlinear growth and to expedite the simulations. Thus, we begin with $\delta=1$ for all models, which in fact would be consistent with turbulent nature of the halo medium.

\subsection{Radiative Cooling Rates}

The key idea of the GC formation model based on the thermal instability, originally suggested by Fall \& Rees (1985), is that the characteristic mass scale of GCs, $M_{c} \sim 10^{6} M_{\odot}$, can be explained by the imprinting of the Jeans mass of the gas clouds at $T=10^{4} \mathrm{~K}$ that have cooled from a hot halo gas in pressure equilibrium. If the clouds were allowed to cool well below $10^{4} \mathrm{~K}$ in a timescale shorter than the free-fall timescale, they would not retain the memory of the imprinted mass scale. Thus, in this work we consider the cases in which the radiative cooling is ineffective below $10^{4} \mathrm{~K}$. If the halo gas had been enriched by the metals from the first objects, or if $\mathrm{H}_{2}$ molecules have formed efficiently via gas-phase reactions, then the gas would have cooled well below $10^{4} \mathrm{~K}$ before the Jeans mass was imprinted (Shapiro \& Kang 1987). Thus, we consider a primordial gas with $\mathrm{H}$ and $\mathrm{He}$ only, and we assume that the formation of $\mathrm{H}_{2}$ molecules is prohibited as a result of UV radiation from central active galactic nuclei or diffuse background radiation (Kang et al. 1990), resulting in zero cooling for $T<10^{4} \mathrm{~K}$.

Here we define the cooling rate as

$$
\Lambda\left(T, n_{\mathrm{H}}\right)=L(T) n_{\mathrm{H}}^{2},
$$

where $\Lambda$ is the energy-loss rate per unit volume and $n_{\mathrm{H}}$ is the number density of hydrogen nuclei. In general, the cooling rate coefficient, $L(T)$, is a function of temperature as well as ionization fractions that can be dependent on the thermal and ionization history of gas. When a hot gas cools from $T>10^{6} \mathrm{~K}$, in particular, it recombines out of ionization equilibrium because the cooling timescale is shorter than the recombination timescale (Shapiro \& Kang 1987). Thus, in order to calculate the nonequilibrium cooling rate accurately, the time-dependent equations for ionization fractions should be solved, which can be computationally expensive. Fortunately, however, the nonequilibrium cooling rate for the gas cooling under the isobaric condition becomes a function of temperature only, if the initial temperature is high enough to ensure the initial ionization equilibrium (e.g., $T>10^{6} \mathrm{~K}$ ) and if only two-body collisional processes are included. In that case, $L(T)$ can be represented 
by a tabulated form as a function of temperature only. We adopt, as our standard cooling model, the nonequilibrium radiative cooling rate for a zero-metallicity, optically thin gas that is calculated by following the nonequilibrium collisional ionization of the gas cooling from $10^{7.5}$ to $10^{4} \mathrm{~K}$ under the isobaric condition (Sutherland \& Dopita 1993). We set $L(T)=0$ for $T<10^{4} \mathrm{~K}$ and, in addition, set the minimum temperature at $T_{\min }=10^{4} \mathrm{~K}$.

In order to explore the effects of different cooling rates, we have also calculated several models with the following cooling rates, in addition to the standard cooling model (NEQm0): (1) the CIEm0 model - the collisional ionization equilibrium cooling for a zero-metallicity gas; and (2) the NEQm1 model - the nonequilibrium cooling rate for a gas with metallicity $Z=0.1 Z_{\odot}$. The NEQm1 model is not consistent with our assumption of zero cooling rate below $T=10^{4} \mathrm{~K}$ but has been included for comparison. Figure 1 shows the cooling rate coefficients for these cooling models.

As the central part of the cloud cools, a steep temperature gradient develops between the cloud and hot background

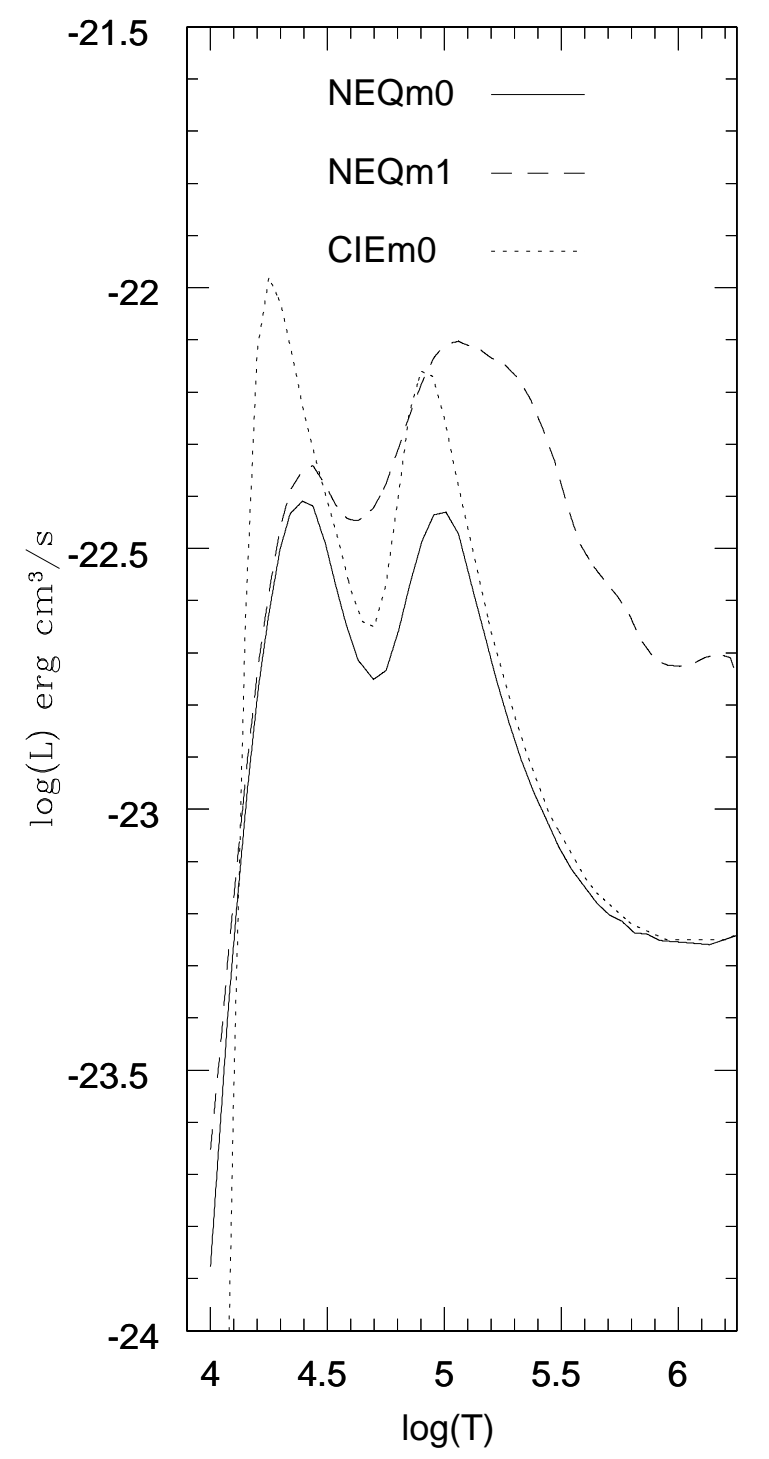

medium and the thermal conduction can become operative there. Brinkman et al. (1990) showed, however, that their simulation results differ by $\leq 3 \%$ when the reduced thermal conductivity (Gray \& Kilkenny 1980) was included. Thus, we ignore the thermal conduction in our calculations, although it may possibly be important.

\subsection{Cooling Time and Length}

We define the cooling time as

$$
t_{\mathrm{cool}}=\frac{\epsilon}{\Lambda}=\frac{n_{\mathrm{tot}} k T}{(\gamma-1) \Lambda}
$$

where $\epsilon=P /(\gamma-1)$ is the internal energy per unit volume and $n_{\text {tot }}$ is the number density of ions and electrons. With the standard NEQm0 cooling model, the cooling time for the background halo gas is

$$
t_{\mathrm{cl}, h}=\left(2.0 \times 10^{7} \mathrm{yr}\right)\left(\frac{n_{h}}{0.1 \mathrm{~cm}^{-3}}\right)^{-1},
$$

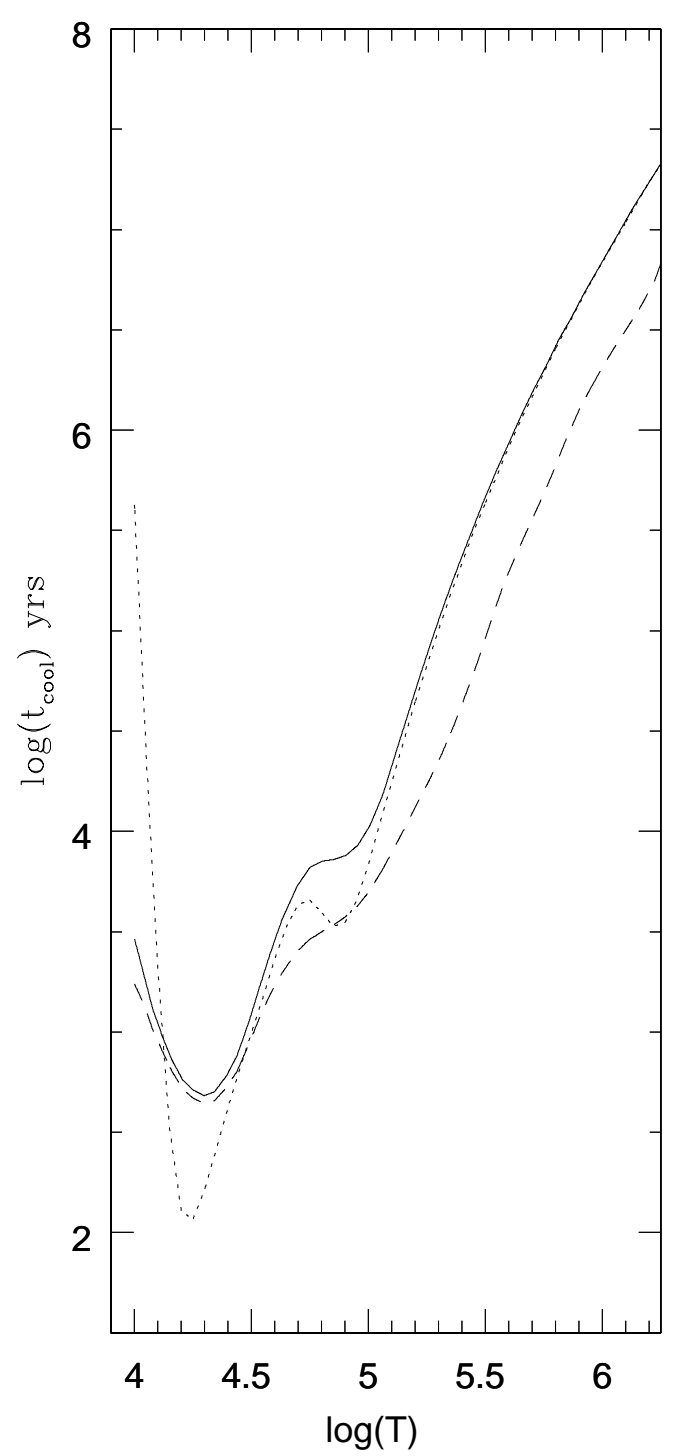

FIG. 1. - Left: Cooling rate coefficient, $L(T)=\Lambda / n_{\mathrm{H}}^{2}$, for the standard cooling model (NEQm0; solid line), the collisional ionization equilibrium cooling model for a zero-metallicity gas (CIEm0; dotted line), and the nonequilibrium cooling model for a gas with metallicity $Z=Z_{\odot} / 10$ (NEQm1; dashed line; see text). Right: Cooling time, $t_{\text {cool }}=\epsilon / \Lambda$, for a gas cooling from $T_{h}=1.7 \times 10^{6} \mathrm{~K}$ with $n_{h}=0.1 \mathrm{~cm}^{-1}$ initially under the isobaric condition. The same line type is used for different cooling models as in the cooling rate coefficient. 
with $T_{h}=1.7 \times 10^{6} \mathrm{~K}$. Figure 1 shows the cooling time for a gas cooling under the isobaric condition (i.e., $n_{\text {tot }} T=$ const) for three cooling models. The cooling time for the gas at the cloud center is given by

$$
t_{\mathrm{cl}, c}=\frac{t_{\mathrm{cl}, h}}{(1+\delta)^{2}}=\frac{t_{\mathrm{cl}, h}}{4},
$$

with $n_{\text {cloud }}=n_{h}(1+\delta), T_{\text {cloud }}=T_{h} /(1+\delta)$, and $\delta=1$. We note that the cloud center cools in a timescale of $\sim 0.5 t_{\mathrm{cl}, h}$, while the background halo cools in $\sim 2 t_{\mathrm{cl}, h}$.

We define the cooling length as the distance over which the sound wave of the hot halo gas travels within one cooling time of the perturbed gas,

$$
l_{\mathrm{cool}}=c_{h} t_{\mathrm{cl}, c}=(1.05 \mathrm{kpc})\left(\frac{n_{h}}{0.1 \mathrm{~cm}^{-3}}\right)^{-1},
$$

where $c_{h}=198 \mathrm{~km} \mathrm{~s}^{-1}$ is the sound speed of the hot gas of $T_{h}=1.7 \times 10^{6} \mathrm{~K}$. The dynamics of radiatively cooling clouds is characterized by the cloud size relative to the cooling length (Fall \& Rees 1985; David et al. 1988; Kang et al. 2000).

The mass contained within a cloud of radius $R_{c}=l_{\text {cool }}$ is

$$
M_{\text {cool }}=\frac{4 \pi}{3} l_{\text {cool }}^{3} \rho_{h}=\left(1.75 \times 10^{7} M_{\odot}\right)\left(\frac{n_{h}}{0.1 \mathrm{~cm}^{-3}}\right)^{-2} .
$$

For $n_{h}=0.1 \mathrm{~cm}^{-3}$, the clouds with the size $R_{c} \sim(0.4-1) l_{\text {cool }}$ have the mass scales of $M_{\text {cloud }} \approx 10^{6}-10^{7} M_{\odot}$ that are relevant for PGCCs. Because of the $n_{h}^{-2}$ dependence, for the background halo density that is much larger or much smaller than our fiducial value, the characteristic cooling mass would be too small or too big, respectively, for the cooled perturbations to become PGCCs.

\subsection{Numerical Method}

The gasdynamical equations for an axisymmetric system in the cylindrical coordinate system, $(R, z, \phi)$, including radiative cooling are written as

$$
\begin{gathered}
\frac{\partial \rho}{\partial t}+\frac{1}{R} \frac{\partial}{\partial R}\left(R \rho u_{R}\right)+\frac{\partial}{\partial z}\left(\rho u_{z}\right)=0, \\
\frac{\partial\left(\rho u_{R}\right)}{\partial t}+\frac{1}{R} \frac{\partial}{\partial R}\left(R \rho u_{R}^{2}\right)+\frac{\partial}{\partial z}\left(\rho u_{R} u_{z}\right)-\frac{\partial P}{\partial R}=0, \\
\frac{\partial\left(\rho u_{z}\right)}{\partial t}+\frac{1}{R} \frac{\partial}{\partial R}\left(R \rho u_{R} u_{z}\right)+\frac{\partial}{\partial z}\left(\rho u_{z}^{2}\right)-\frac{\partial P}{\partial z}=0, \\
\frac{\partial(\rho e)}{\partial t}+\frac{1}{R} \frac{\partial}{\partial R}\left[R u_{R}(\rho e+P)\right]+\frac{\partial}{\partial z}\left[u_{z}(\rho e+P)\right]=-\Lambda,
\end{gathered}
$$

where $e=\epsilon / \rho+\frac{1}{2} u^{2}$ is the total energy of the gas per unit mass, $R=\left(x^{2}+y^{2}\right)^{1 / 2}, u=u_{R}^{2}+u_{z}^{2}$, and the rest of the variables have their usual meanings.

To solve the hydrodynamic part, we have used a Eulerian, grid-based hydrodynamics code based on the total variation diminishing (TVD) scheme (Ryu et al. 1993). The cylindrical geometry version has been used for two-dimensional simulations. For one-dimensional comparison simulations, the spherical geometry version has been used. The TVD scheme solves a hyperbolic system of gasdynamical conservation equations with a second-order accuracy. Multidimensionality is handled by the Strang-type dimensional splitting (Strang 1968).
After completion of the hydrodynamic part updating hydrodynamical quantities from the time step $t^{n}$ to $t^{n+1}$, radiative cooling is applied to the thermal energy as a separate part. If we were to update the thermal energy density by the explicit scheme

$$
\epsilon^{n+1}=\epsilon^{n}-\Lambda \Delta t=\epsilon^{n}\left(1-\frac{\Delta t}{t_{\mathrm{cool}}^{n+1 / 2}}\right),
$$

the time step size should be smaller than the cooling time, that is, $\Delta t<t_{\mathrm{cool}}^{n+1 / 2}$. Here $t_{\mathrm{cool}}^{n+1 / 2}$ is estimated from the timeaveraged hydrodynamic quantities, $\left(q^{n}+q^{n+1}\right) / 2$. This explicit integration scheme would be extremely expensive, when the cooling timescale is much shorter than the hydrodynamical timescale. For that reason, we rewrite the cooling part of the thermal energy equation as

$$
\frac{d \ln (\epsilon)}{d t}=-\frac{\Lambda}{\epsilon}=-\frac{1}{t_{\mathrm{cool}}} .
$$

In our numerical code we integrate this equation as

$$
\epsilon^{n+1}=\epsilon^{n} \exp \left(-\frac{\Delta t_{h}}{t_{\mathrm{cool}}^{n+1 / 2}}\right),
$$

assuming that $t_{\text {cool }}$ is constant over the hydrodynamic timescale $\Delta t_{h}$ (e.g., LeVeque 1997). Strictly speaking, equation (14) converges to equation (12) only when $\Delta t_{h}$ is much smaller than $t_{\text {cool }}$. When $\Delta t_{h} \gtrsim t_{\text {cool }}^{n+1 / 2}$, however, the gas quickly looses most of the thermal energy via radiation and the temperature approaches the specified minimum value $\left(T_{\min }\right)$ during one hydrodynamic time step, if we were to integrate equation (12) with a small time step size of $t_{\text {cool }}$ for many steps. However, this behavior is emulated reasonably well by integrating equation (14) with $\Delta t_{h}$ for one step, which effectively lowers the gas temperature to $T_{\min }$. Thus, the end results of both integration schemes would be qualitatively similar, although they could be quantitatively somewhat different. Especially at different spatial grid resolutions, the detailed thermal history of the gas could be different during the cooling phase, but once cooled the final structure should be roughly similar. With this integration scheme, gas cooling can be followed, although approximately, with the hydrodynamic time steps, even when the cooling timescale is shorter than the hydrodynamic timescale.

Our simulations start at $t=0$ with the clouds at rest in pressure equilibrium $\left(P_{\text {halo }}=P_{\text {cloud }}\right)$ and cease at $t=2 t_{\text {cool }}$ when the background gas has cooled to $10^{4} \mathrm{~K}$. The standard mirror condition has been used for the reflecting boundaries at $R=0$ and $z=0$, while it has been assumed that flows are continuous across the outer boundaries. We have used only one quadrant of the cylindrical coordinate system.

In our simulations the clouds cool and collapse as a result of the compression by the background pressure to the size that is $1 / 10$ or so of the initial cloud radii. In order to study the detailed structure of the collapsed clouds, we have devised a special grid that consists of an inner fine zone with uniform grid spacing and an outer coarse zone with expanding grid spacing. The inner zone has $1000^{2}$ uniform cells with $\Delta R=\Delta z=L_{\text {fine }} / 1000$, where $L_{\text {fine }}=1.6 l_{\text {cool }}$. The outer zone has $1240^{2}-1000^{2}$ cells covering the rest of the simulated region outside the inner fine zone, with the cell spacing that increases outward as $\Delta R^{i} / \Delta R^{i-1}=$ 
TABLE 1

Initial Parameters for Model Clouds

\begin{tabular}{|c|c|c|c|c|c|c|c|c|}
\hline Model & $S_{i}$ & $R_{c, R} / l_{\text {cool }}$ & $R_{c, z} / l_{\text {cool }}$ & $\begin{array}{l}R_{c, \text { max }} \\
(\mathrm{kpc})\end{array}$ & $\begin{array}{c}M_{\text {cloud }} \\
\left(\times 10^{6} M_{\odot}\right)\end{array}$ & $\begin{array}{c}M_{4}^{\mathrm{a}} \\
\left(\times 10^{6} M_{\odot}\right)\end{array}$ & $\begin{array}{c}M_{\mathrm{J}}^{\mathrm{a}} \\
\left(\times 10^{6} M_{\odot}\right)\end{array}$ & $\begin{array}{c}\left\langle\rho_{4}\right\rangle^{\mathrm{a}} \\
{\left[\left(\times 2.34 \times 10^{-24} \mathrm{~g}\right) n_{h}\right]}\end{array}$ \\
\hline $\mathrm{S} 11 \ldots \ldots \ldots \ldots \ldots \ldots$ & 1.0 & 0.4 & 0.4 & 0.421 & 1.489 & 1.156 & 45.222 & 96.125 \\
\hline $\mathrm{S} 12 \ldots \ldots$ & 0.5 & 0.2 & 0.4 & 0.421 & 0.376 & 0.455 & 47.744 & 86.236 \\
\hline $\mathrm{S} 21 \ldots \ldots \ldots \ldots \ldots \ldots$ & 2.0 & 0.4 & 0.2 & 0.421 & 0.745 & 0.856 & 49.797 & 79.271 \\
\hline M11 ................. & 1.0 & 0.8 & 0.8 & 0.842 & 11.858 & 8.940 & 32.831 & 182.367 \\
\hline M12 ................. & 0.5 & 0.4 & 0.8 & 0.842 & 2.979 & 2.687 & 47.934 & 85.557 \\
\hline M56 .................. & $5 / 6$ & 0.67 & 0.8 & 0.842 & 8.243 & 8.059 & 65.272 & 46.139 \\
\hline M65 .................. & $6 / 5$ & 0.8 & 0.67 & 0.842 & 9.881 & 7.935 & 65.407 & 45.949 \\
\hline M21 ............... & 2.0 & 0.8 & 0.4 & 0.842 & 5.929 & 5.928 & 50.757 & 76.304 \\
\hline L11 ................. & 1.0 & 1.6 & 1.6 & 1.68 & 94.622 & 71.112 & 58.688 & 57.074 \\
\hline L12 ................. & 0.5 & 0.8 & 1.6 & 1.68 & 23.715 & 19.752 & 40.654 & 118.941 \\
\hline L56 ................. & $5 / 6$ & 1.33 & 1.6 & 1.68 & 56.741 & 55.689 & 39.360 & 126.890 \\
\hline L65 ................. & $6 / 5$ & 1.6 & 1.33 & 1.68 & 78.852 & 61.853 & 45.436 & 95.218 \\
\hline L21 ................. & 2.0 & 1.6 & 0.8 & 1.68 & 47.310 & 40.701 & 35.067 & 159.857 \\
\hline EL56 ................ & $5 / 6$ & 1.33 & 1.6 & 1.68 & 56.741 & 64.934 & 6.653 & 855.259 \\
\hline EL65 ................ & $6 / 5$ & 1.6 & 1.33 & 1.68 & 78.852 & 65.678 & 10.307 & 356.416 \\
\hline ML56............... & $5 / 6$ & 1.33 & 1.6 & 1.68 & 56.741 & 92.528 & 46.025 & 93.201 \\
\hline ML65................ & $6 / 5$ & 1.6 & 1.33 & 1.68 & 78.852 & 105.559 & 35.390 & 157.635 \\
\hline X11 ................. & 1.0 & 3.2 & 3.2 & 3.36 & 756.981 & 571.840 & 193.961 & 5.225 \\
\hline $\mathrm{X} 12 \ldots \ldots \ldots \ldots \ldots$ & 0.5 & 1.6 & 3.2 & 3.36 & 189.721 & 157.458 & 47.869 & 85.789 \\
\hline X21 „................. & 2.0 & 3.2 & 1.6 & 3.36 & 378.481 & 286.662 & 65.393 & 45.969 \\
\hline
\end{tabular}

a Calculated at $t=1.4 t_{\mathrm{cl}, h}$.

$\Delta z^{i} / \Delta z^{i-1}=1.05$ for $i=1001, \ldots, 1240$. With the expanding grid the outer boundaries are located far away from the central cloud where most activity occurs.

The physical variables are expressed in units of the following normalization both in the numerical code and in the plots presented below: $t_{0}=t_{\mathrm{cl}, h}=2.0 \times 10^{7} \mathrm{yr} ; r_{0}=$ $l_{\text {cool }}=1.05 \mathrm{kpc} ; \quad u_{0}=l_{\text {cool }} / t_{\mathrm{cl}, h}=50.8 \quad \mathrm{~km} \mathrm{~s}^{-1} ; \quad \rho_{0}=$ $\left(2.34 \times 10^{-24} \mathrm{~g}\right) n_{h} ; P_{0}=\rho_{0} u_{0}^{2}=6.03 \times 10^{-12} \mathrm{ergs} \mathrm{cm}^{-3}$.

\subsection{Shape Parameter}

In order to quantify how the cloud shape evolves, we define the "shape parameter" as

$$
S=\frac{R_{e, R}}{R_{e, z}},
$$

where $R_{e, R}$ and $R_{e, z}$ are the "effective" radii along the $R$ and $z$-axes, respectively. Here the "effective" radius is defined as the radius where the gas density decreases to half of the central density, that is, $\rho\left(R_{e}\right)=\frac{1}{2} \rho_{c}$. The initial values of the shape parameter for models considered are $S_{i}=1$ for spherical clouds, $S_{i}=\frac{5}{6}$ and $\frac{1}{2}$ for prolate clouds, and $S_{i}=6 / 5$ and 2 for oblate clouds. The second column of Table 1 shows the initial values of the shape parameter for each model.

\section{SIMULATION RESULTS}

\subsection{Spherical Symmetric Calculations}

We have first calculated spherical symmetric collapses with both one- and two-dimensional codes, in order to show their behavior and to compare it with that of nonspherical collapses, as well as to test the performance of the twodimensional code. According to our previous onedimensional simulations, which employed a different code (the PPM code; Kang et al. 2000), the evolution of spherical clouds collapsing via the thermal instability can be classified by the cloud size as follows: $(1)\left(R_{c} / l_{\text {cool }}\right)<0.2$, the isobaric compression regime, (2) $0.2 \lesssim\left(R_{c} / l_{\text {cool }}\right) \lesssim 1-1.5$, the supersonic compression regime, and (3) $\left(R_{c} / l_{\text {cool }}\right) \gtrsim 1-1.5$, the isochoric cooling regime.

The following four different sizes have been considered in our spherical symmetric calculations: three supersonic cloud models, S11 with $R_{c}=0.4 l_{\text {cool }}$, M11 with $R_{c}=0.8 l_{\text {cool }}$, and L11 with $R_{c}=1.6 l_{\mathrm{cool}}$, and one isochoric cloud model, X11 with $R_{c}=3.2 l_{\text {cool }}$ (see Table 1 ). The largest cloud model, $\mathrm{X} 11$, has a cloud mass too large for PGCCs, but it has been included for comparison. We first note that the results from the current one-dimensional simulations are consistent with those from our previous simulations in Kang et al. (2000), although different numerical codes are used. While the CIEm0 cooling model was adopted in Kang et al. (2000), here we have adopted the standard NEQm0 cooling model, which has lower peaks around both the $\mathrm{H}$ and $\mathrm{He}$ Ly $\alpha$ line emissions (see Fig. 1). As a result, gas cools less rapidly and central density increases to lower values in the current simulations.

The results from two-dimensional simulations of spherical collapses are mostly similar to those from onedimensional simulations. Figure 2 shows the distributions of the gas density, the velocities in the $R$ - and $z$-directions, and the pressure along the diagonal line of $R=z$ from the two-dimensional simulation for the L11 model with $R_{c}=1.6 l_{\text {cool }}$. The distributions are sampled at $t / t_{\mathrm{cl}, h}=0.2$ (solid line), 0.6 (dotted line), 1.0 (dashed line), 1.4 (longdashed line), and 1.8 (dot-dashed line). As the central gas cools and loses pressure, the high-pressure background compresses the cloud and induces an infall flow for $t / t_{\mathrm{cl}, h} \lesssim 0.6$. The flow velocity increases up to $\left(u / c_{h}\right) \sim 0.36$, which is much larger than the sound speed of the cooled gas, $\left(c_{c} / c_{h}\right) \sim 0.08$. Hence, the accretion flow is supersonic. After $t=0.6 t_{\mathrm{cl}, h}$, the infall flow bounces back as an accretion shock and the shock moves out. Then, the cloud expands outward slowly as a result of the high pressure at 
$\left[R_{c}=1.61_{\text {cool }}\right.$, Spherical Symmetric $]$
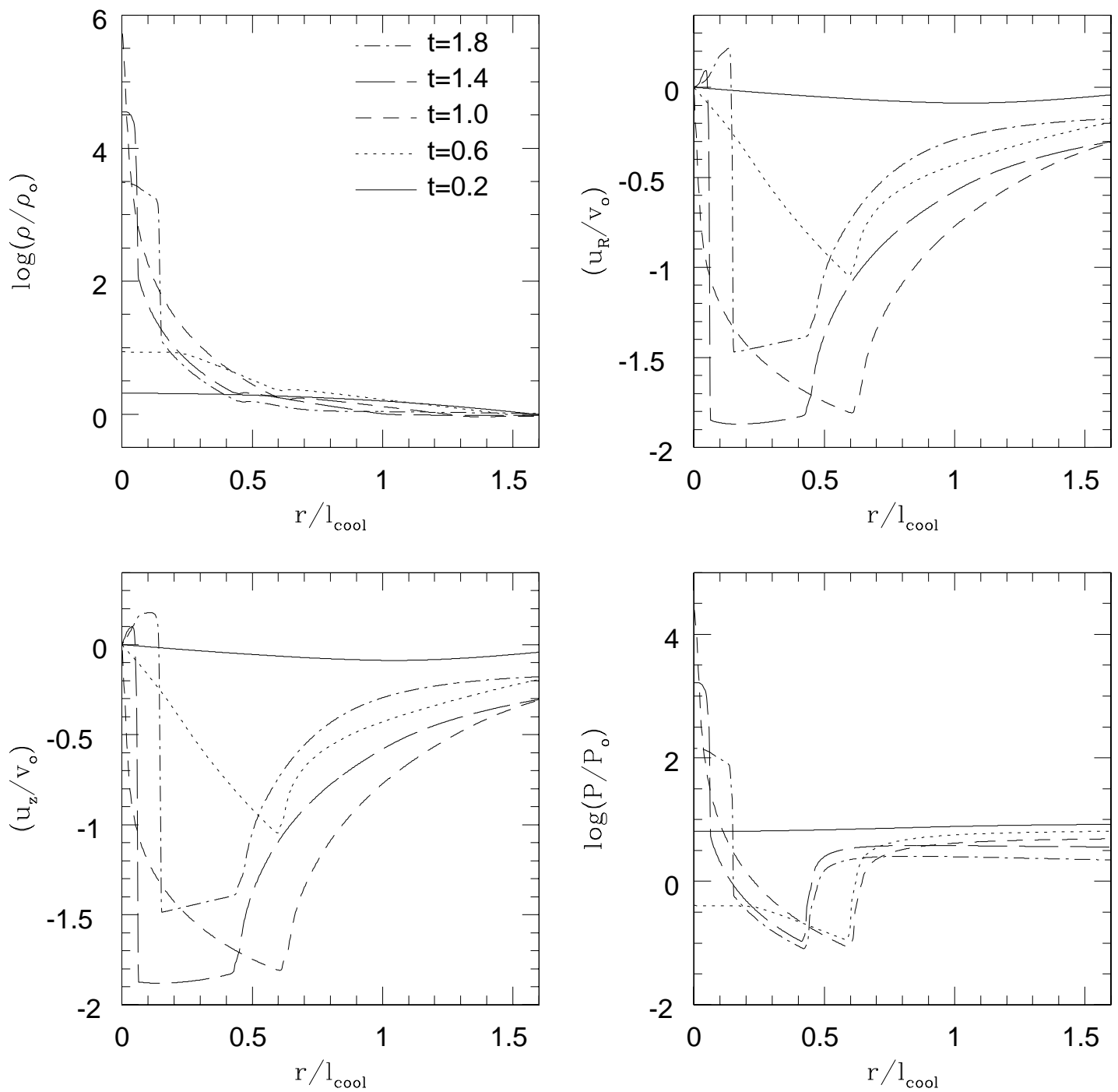

Fig. 2.-Time evolution of the gas density, $\rho$, the velocities, $u_{R}, u_{z}$, and the pressure, $P$, at $t / t_{\mathrm{cl}, h}=0.2$ (solid line), 0.6 (dotted line), 1.0 (dashed line), 1.4 (longdashed line), and 1.8 (dot-dashed line) for the spherical cloud with $R_{c}=1.6 l_{\mathrm{cool}}$ (the L11 model). Distributions along the diagonal line of $R=z$ in the twodimensional box are shown, as a function of the radial distance $r=\left(R^{2}+z^{2}\right)^{1 / 2}$.

the central region. One can see that the central density increases up to $\rho_{c} / \rho_{o} \sim 10^{6}$ just before the shock bounces back and then decreases to $\rho_{c} / \rho_{o} \sim 10^{3.5}$ as the collapsed cloud expands. The shape of the collapsed clouds from twodimensional simulations becomes slightly rectangular, especially for the models with small sizes. The deviation from the spherical symmetry, measured with the shape parameter $(S-1)$, ranges from less than $1 \%$ (in the X11 model) to $\sim 20 \%$ (in the S11 model).

\subsection{Two-dimensional Axisymmetric Calculations}

For the nonspherical, axisymmetric clouds, four different sizes are considered, as for the spherical clouds: $R_{c, \text { max }}=0.4 l_{\text {cool }}$ for small clouds, $R_{c, \text { max }}=0.8 l_{\text {cool }}$ for medium-size clouds, $R_{c, \max }=1.6 l_{\text {cool }}$ for large clouds, and $R_{c, \max }=3.2 l_{\text {cool }}$ for very large clouds. Here $R_{c, \max }=\max \left(R_{c, R}, R_{c, z}\right)$. The initial parameters of the model clouds considered are summarized in Table 1.
Figures 3 and 4 show the time evolution of the density distribution of the large prolate cloud, the L56 model with $S_{i}=\frac{5}{6}$, and the large oblate cloud, the L65 model with $S_{i}=6 / 5$, respectively. Figure 5 shows the flow velocity field superimposed on the density contour maps of the L56 (lefthand panels) and L65 (right-hand panels) models at $t=0.8 t_{\mathrm{cl}, h}$ (top panels) and $t=1.6 t_{\mathrm{cl}, h}$ (bottom panels). As gas cools catastrophically, the clouds implode near the center and supersonic anisotropic accretion flow is induced. The compression continues until $t \approx 0.8 t_{\mathrm{cl}, h}$ when infall flow is reflected at the center and an accretion shock forms. This "shock formation time" depends on the initial cloud size, as the infall flow turns around later in larger clouds. At the shock formation time, the infall flow field is strongly anisotropic, preferentially parallel to the $z=0$ plane for the prolate cloud and parallel to the $z$-axis for the oblate cloud. As a result, the degree of prolateness or oblateness is enhanced. Thus, the prolate cloud collapses to a very thin rod shape, while the oblate cloud collapses to a flat disk, at $t \approx 0.8 t_{\mathrm{cl}, h}$ in the L56 and L65 models. The central density peaks at the 
[L56:Prolate, $\mathrm{S}=1 / 1.2]$
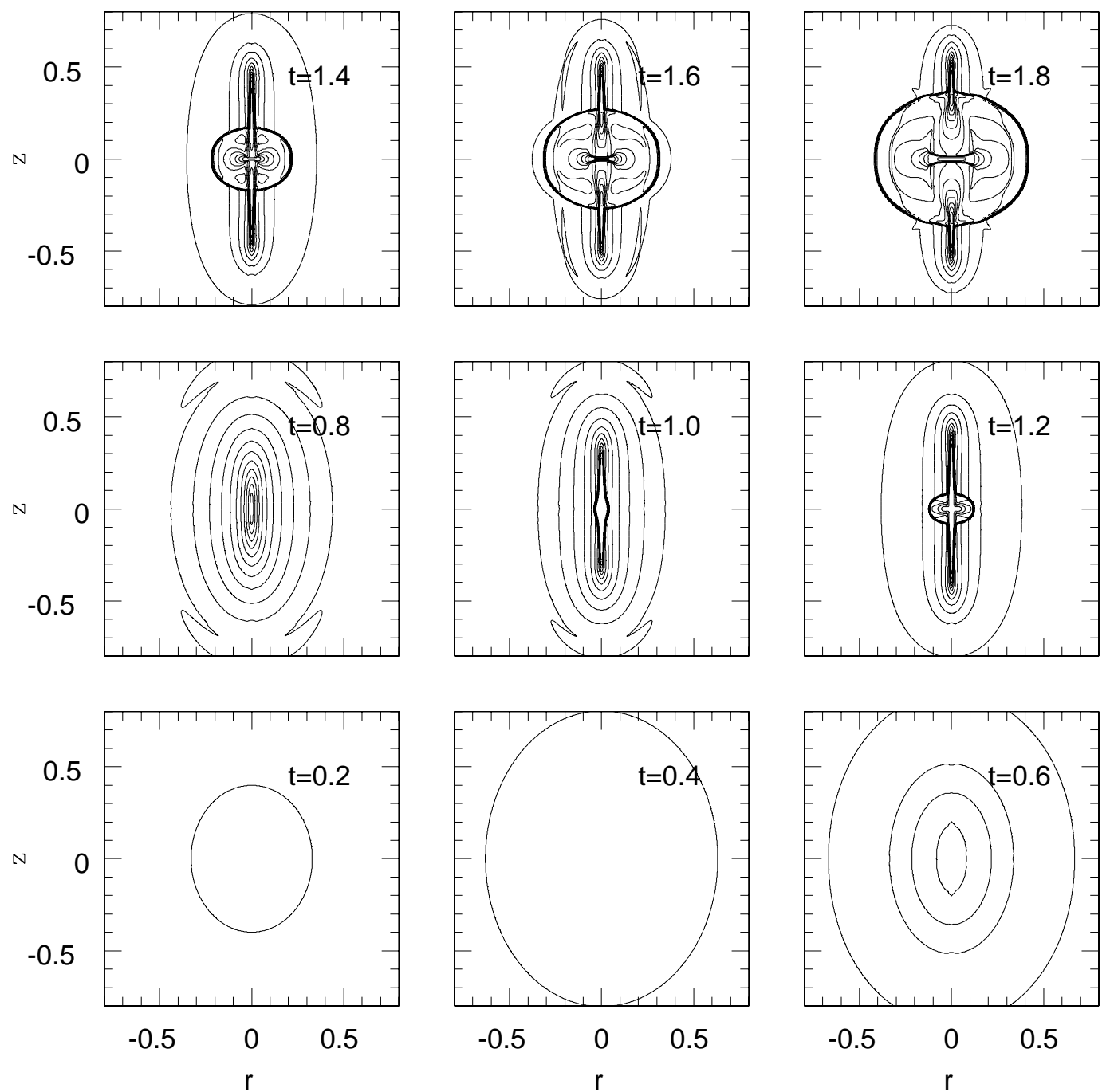

FIG. 3. - Time evolution of the density distribution in the prolate cloud with $S=\frac{5}{6}$ and $R_{c, \max }=1.6 l_{\text {cool }}$ (the model L56). The contour levels for $\left(\rho / \rho_{0}\right)=2^{i}$ $(i=0,1, \ldots, 10)$ are shown. Full images are generated from the simulation data covering one quadrant of the whole space by using the reflection symmetry along the $z=0$ plane and along the $z$-axis.

time of shock formation and then slowly decreases afterward. After that time, the accretion shock halts the infall flow, as shown in the bottom panels of Figure 5. Inside the accretion shock, there exist residual infall motions with the preferred direction different from that of early accretion before $t=0.8 t_{\mathrm{cl}, h}$, that is, along the $z$-axis for the prolate model and along the $z=0$ plane for the oblate model. So now the contraction proceeds along the $z$-axis toward the center, which in turn generates the radial outflow along the $z=0$ plane inside the cooled cloud in the prolate model. In the oblate model, on the other hand, the contraction occurs along the $z=0$ plane toward the center and induces the bipolar outflow along the $z$-axis. This "secondary" contraction leads to a central bulge that has a shape reverse to the initial shape of the cloud. Thus, the prolate cloud results in a structure that consists of a cigar-shaped outer component and a central oblate component. On the other hand, the oblate cloud collapsed to a structure of a pancakeshaped outer component and a central prolate component.
In the two-dimensional simulation of Brinkman et al. (1990), they considered an oblate cloud $\left(S_{i} \approx 2.2\right)$ with small density contrast, $\delta=0.1$, in a background with $T_{h}=10^{7} \mathrm{~K}$ and $n_{h}=0.1 \mathrm{~cm}^{-3}$, hotter than ours. They showed that the cloud collapses to a flat structure with density enhancement of $\sim 100$ at $t \sim 3$ in units of their cooling time. Because their simulation started with a small $\delta$, it ended just after the formation of a nonlinear pancake-like structure that corresponds to the structure at $t \sim 0.9 t_{\mathrm{cl}, h}$ in our simulation (see Fig. 4). As a result, they did not see the development of the inner central prolate condensation. Whether collapses end at the cigar/pancake formation stage or they continue to form the inner oblate/prolate components depends mainly on the cloud size ratio, $R_{c} / l_{\text {cool }}$, and the contrast of cooling times, $t_{\mathrm{cl}, c} / t_{\mathrm{cl}, h}$. Thus, these two parameters along the initial shape parameter, $S_{i}$, determine the dynamics and mass distribution of the collapsed clouds.

Figures 6 and 7 show the evolutionary sequences for the medium-size prolate cloud, the M12 model with $S_{i}=\frac{1}{2}$, and 
[L65:Oblate, $\mathrm{S}=1.2]$
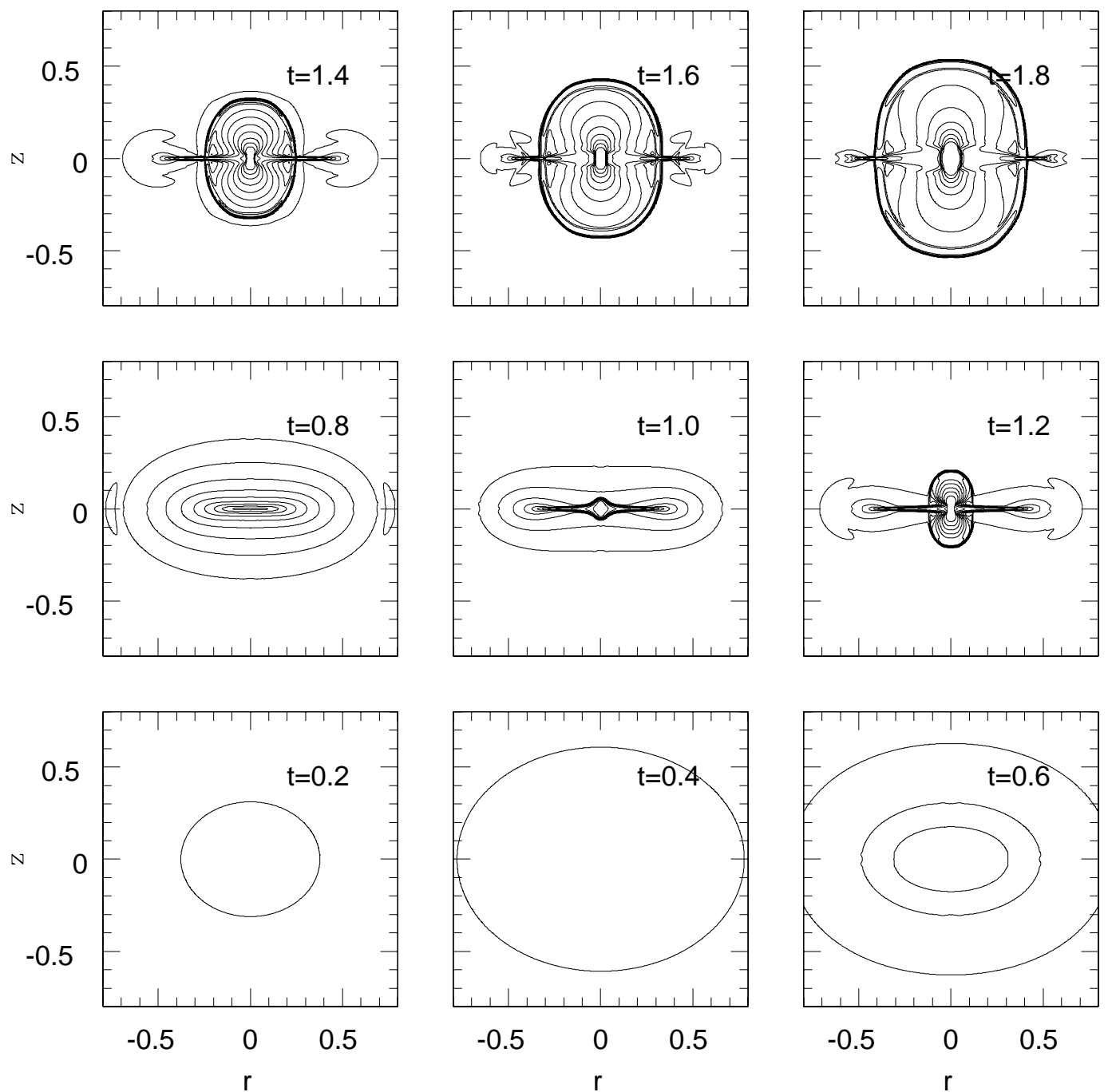

FIG. 4.-Same as in Fig. 3, except for the oblate cloud with $S=6 / 5$ and $R_{c, \max }=1.6 l_{\text {cool }}$ (the model L65)

the medium-size oblate cloud, the M21 model with $S_{i}=2$, respectively. Because of a higher degree of initial nonsphericity, these models display much stronger shape instability than the L56 and L65 models. The shock formation time is $t \approx 0.6 t_{\mathrm{cl}, h}$, slightly earlier than that for the L56 and L65 models because of smaller sizes. The inner components due to the secondary contraction grow less significantly, compared to those of the L56 and L65 models.

To illustrate how the initial shape parameter and cloud size affect the evolution, we plot in Figure 8 the density contour maps at $t=1.6 t_{\mathrm{cl}, h}$ for the models listed in Table 1 that adopt the standard cooling. For all models, the regions bounded by $\left(-0.5 R_{c, R}, 0.5 R_{c, R}\right) \times\left(-0.5 R_{c, z}, 0.5 R_{c, z}\right)$ are shown. Thus, for example, $(-0.4,0.4) \times(-0.4,0.4)$ in units of $l_{\text {cool }}$ is shown for the $\mathrm{M}$ models. Except in the very large clouds (the X models), the shape reversal is observed in the central part of cooled clouds, that is, the formation of the inner oblate (prolate) component in the prolate (oblate) models. Spherical models are shown to demonstrate how well the spherical symmetry is conserved in two-dimensional simulations ( $\S 3.1)$. It is interesting to see that the collapses of the clouds with initially even $20 \%$ deviation from the spherical symmetry (i.e., M56, M65, L56, and L65) are very different from the spherical collapses. For a given value of $S_{i}$, the models with smaller cloud sizes have more significant inner components, since the shock formation occurs earlier. For a given cloud size, on the other hand, the models with $S_{i}$ values closer to 1 form more significant inner components. In the very large cloud models, the sound crossing time along the long axis $\left(R_{c, \max }=3.2 l_{\text {cool }}\right)$ is much longer than the cooling time. Hence, the spherical cloud (the X11 model) cools and collapses without forming an accretion shock. However, the X12 and X21 models have the minor-axis radius, $R_{c, \text { min }}=1.6 l_{\text {cool }}$, so they are able to collapse supersonically along the minor axis, forming an accretion shock at $t \approx t_{\mathrm{cl}, h}$. As a result, by $t=1.6 t_{\mathrm{cl}, h}$ the prolate cloud (the X12 model) has been developed into a rod with the central density $\rho_{c} / \rho_{h} \sim 10^{2.7}$, while the oblate cloud (the X21 model) has been developed into a pancake with $\rho_{c} / \rho_{h} \sim 10^{2.3}$. As in the simulation by Brinkman et al. 
[vfield : L56(Prolate) vs L65(Oblate)]
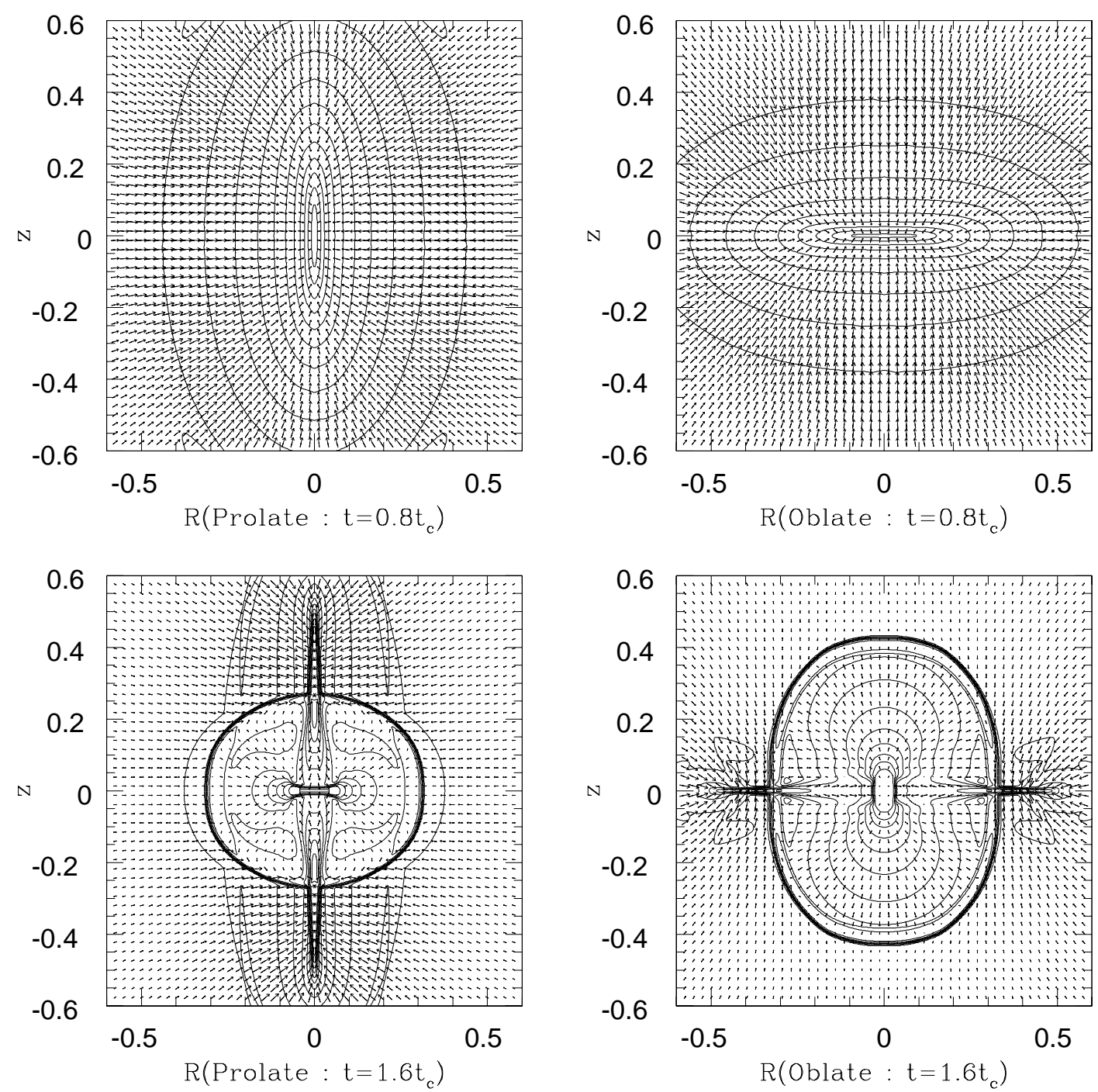

Fig. 5.-Velocity field overlapped on the density contour map for the prolate model L56 (left-hand panels) and the oblate model L65 (right-hand panels) at $t=0.8 t_{\mathrm{cl}, h}$ (top panels) and $t=1.6 t_{\mathrm{cl}, h}$ (bottom panels).

(1990), the inner central components do not display the shape reversal by the time $t \approx 1.6 t_{\mathrm{cl}, h}$ in these models. If we adopt a higher initial density contrast or keep the background gas at constant pressure, however, even the $\mathrm{X}$ models could have developed the inner central components with the reversed shape.

In order to look at the density enhancement in a quantitative way, we present in Figure 9 the density line profiles of the collapsed clouds at $t / t_{\mathrm{cl}, h}=1.8$ for the initially prolate (left-hand panels) and oblate (right-hand panels) clouds. The dotted (dashed) lines show the profiles along the $R$-axis ( $z$ axis), while the solid lines show the profiles of the twodimensional spherical models with the same size (i.e., the S11-X11 models) along the diagonal line of $R=z$ as a function of the radial distance $r=\left(R^{2}+z^{2}\right)^{1 / 2}$. In the prolate models the dashed lines reveal the thin rod-shaped component along the $z$-axis, while the dotted lines show the inner oblate component along the $R$-axis, except in the X12 model where the inner oblate component does not form. In the oblate models, on the other hand, the dotted lines show the flat pancake-shaped component along the $R$-axis, while the dashed lines reveal the inner prolate component along the $z$ axis, except in the X21 model. Once again the shape reversal is observed in the central region $\left[r \lesssim(0.1-0.2) l_{\text {cool }}\right]$ of the cooled clouds, except in the $\mathrm{X}$ models. The outer rodshaped component in the prolate models and the outer pancake-shaped component in the oblate models have density close to the isobaric ratio, $\rho_{\text {cool }} / \rho_{\mathrm{h}} \approx\left(T_{h} \mu_{c} / T_{c} \mu_{h}\right) \approx 10^{2.5}$. The inner oblate/prolate components have the mean density somewhat higher $\left(\rho_{\text {cool }} / \rho_{h} \approx 10^{2.5}-10^{3.5}\right)$ in the $\mathrm{M}$ and $\mathrm{L}$ models. As mentioned before, the central density peaks at the time of shock formation and then decreases afterward as the clouds expand, so the density distribution changes in time. If we compare the central density of different models at a given time after the inner shape reversal appears, the oblate models have higher values than the prolate models, except in the $\mathrm{X}$ models.

Figure 10 shows the evolution of the shape parameter, $S$, for the models with $S_{i}=\frac{1}{2}$ (prolate) and 2 (oblate). During the first "shape instability" stage, the shape parameter is 
[M12:Prolate,S=0.5]
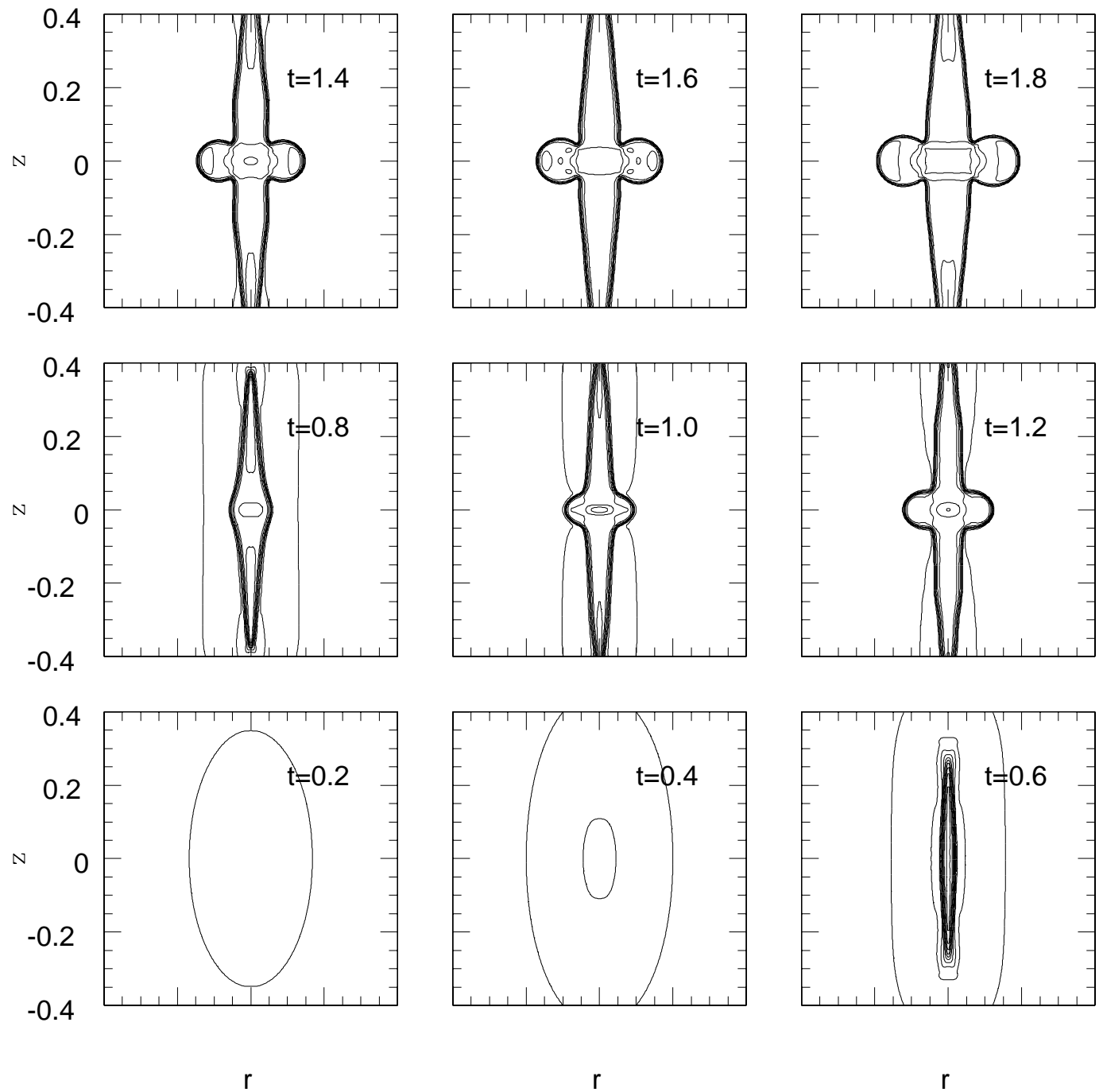

FIG. 6.-Same as in Fig. 3, except for the prolate cloud with $S=\frac{1}{2}$ and $R_{c, \max }=0.8 l_{\text {cool }}$ (the model M12)

determined by the cigar-shaped component or the pancakeshaped component. During the "secondary infall" stage after the shock formation, however, it represents the shape of the inner components in most models (except in the S12 model). In the oblate clouds (right-hand panels), as the clouds collapse to flat pancakes, the value of $S$ increases to $\gg 1$ until the shock formation time. Such time when $S$ reaches the maximum values scales as the sound crossing time $\left(t_{\mathrm{sc}}=R_{c, \min } / c_{h}\right)$, which is proportional to the cloud size. Afterward, the value of $S$ decreases, and it becomes smaller than 1 as a result of the formation of the inner prolate components, except in the model X21. In the prolate clouds (left-hand panels), the value of $S$ decreases to $\ll 1$ as the clouds collapse to thin rod shapes. After the shock formation the value of $S$ increases, and it becomes greater than 1 in the M12 and L12 models as the inner oblate components grow. In the small S12 model, the inner oblate component and the outer cigar-shaped component have similar density, so the effective radius along the $z$-axis represents the length of the cigar component rather than the radius of the inner oblate component, resulting in $S=R_{e, R} / R_{e, z}<1$. For this model, we have also calculated the second shape parameter $S_{100}$, which is defined as the ratio of the radii where $\rho\left(R_{100}\right)=100 \rho_{h}$. As shown in Figure 10, this second shape parameter becomes $S_{100}>1$ at $t \geq 1.6 t_{\mathrm{cl}, h}$, indicating that the inner oblate component indeed forms in the S12 model. In the $\mathrm{X}$ models, $S$ remains either less than 1 or greater than 1 , as expected.

\subsection{Cloud Mass and Mean Density}

In the Fall and Rees model for the formation of PGCCs where the thermal instability is assumed to proceed quasistatically, the "critical mass" defined for an isothermal sphere confined by an external pressure (McCrea 1957)

$$
M_{\mathrm{cr}}=1.18\left(\frac{k T_{c}}{\mu m_{\mathrm{H}}}\right)^{2} G^{-3 / 2} p_{h}^{-1 / 2}
$$

was adopted as the minimum mass for gravitationally unstable clouds. For $T_{c}=10^{4} \mathrm{~K}$ and $p_{h}=3.28 \times 10^{-11} \mathrm{dyn}$ $\mathrm{cm}^{-2}, M_{\mathrm{cr}}=2.8 \times 10^{6} M_{\odot}$. However, this simple picture 
[M21:Oblate,S=2.0]
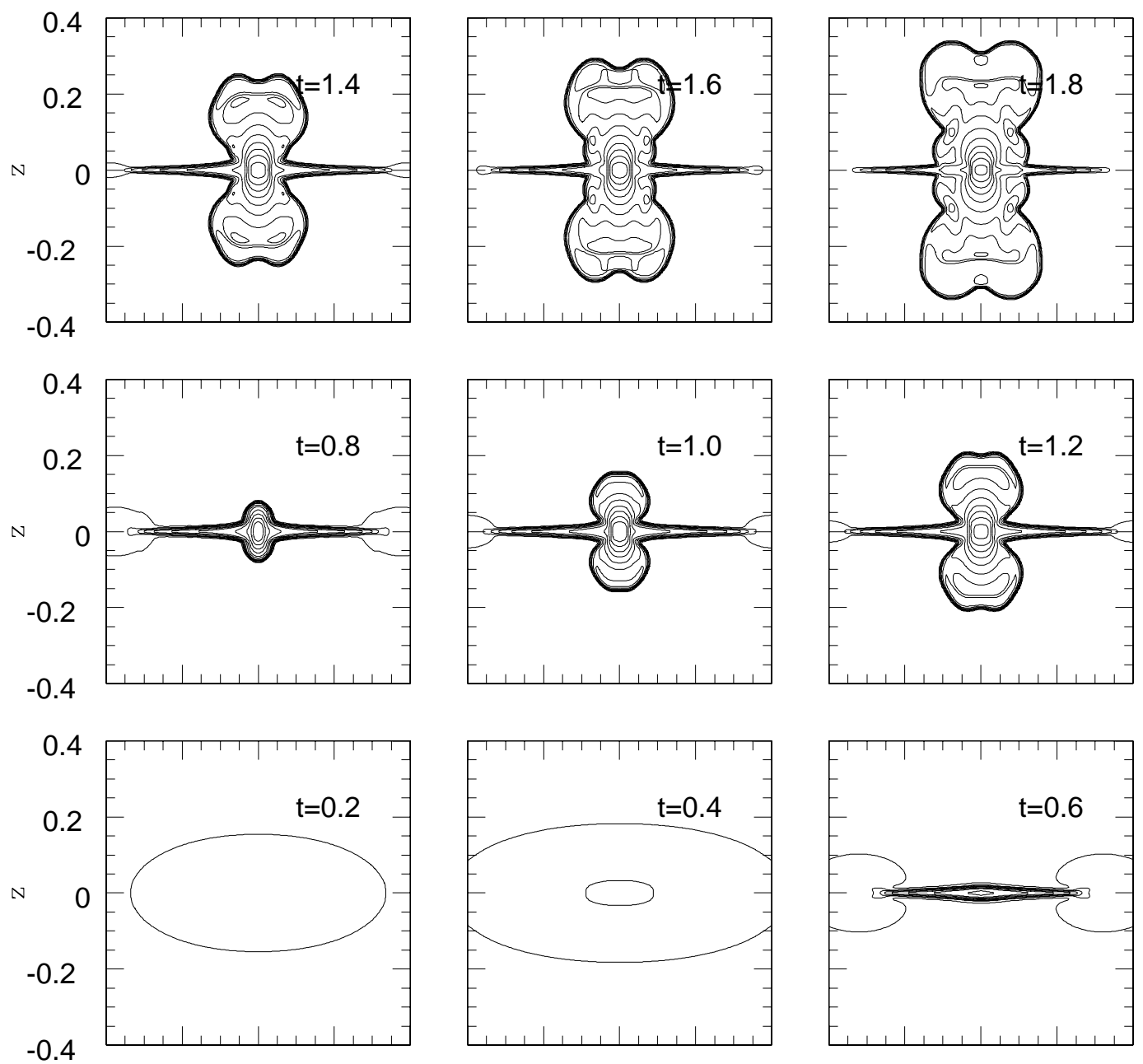

$r$

$r$

r

FIG. 7.- Same as in Fig. 3, except for the oblate cloud with $S=2 / 1$ and $R_{c, \max }=0.8 l_{\text {cool }}$ (the model M21)

should be modified for the following reasons: (1) the collapse is not quasi-static and the infall flows can become supersonic, so the compressed clouds are bound by the ram pressure of the infall flows rather than the background pressure; (2) the cooled compressed clouds (PGCCs) may have turbulent velocity fields (see Fig. 5); and (3) the mass distribution of PGCCs can be very complex, rather than spherical (see Figs. 3-8).

As an effort to obtain a better estimation of the PGCC mass, we have estimated the Jeans mass of the cooled gas in our simulations as follows. First, we have calculated the mass of the gas with $T=10^{4} \mathrm{~K}, M_{4}$. The right-hand panels of Figure 11 show how $M_{4}$ increases with time in our models. Most of the cloud gas cools to $10^{4} \mathrm{~K}$ by $t=1.4 t_{\mathrm{cl}, h}$, so by this time $M_{4}$ becomes comparable to the initial cloud mass, $M_{\text {cloud }}$. We present the values of $M_{4}$ at $t=1.4 t_{\mathrm{cl}, h}$ in the fifth column of Table 1. Next, from the mass and volume of the cells with $T=10^{4} \mathrm{~K}$, we have calculated the mean density of the central region, $\left\langle\rho_{4}\right\rangle$. The values of $\left\langle\rho_{4}\right\rangle$ are shown in the left-hand panels of Figure 11. Although the central density can increase to values much higher than the isobaric compression ratio of $10^{2.5}$, the mean density, $\rho_{4}$, increases only up to that ratio and then decreases, as the background gas cools and the cooled cloud expands. The values of $\left\langle\rho_{4}\right\rangle$ at $t=1.4 t_{\mathrm{cl}, h}$ are listed in the last column of Table 1 .

Finally, although it may not be a good approximation either because of the reasons listed above, we have computed the Jeans mass of an isothermal uniform sphere with temperature $T_{c}$ and density $\rho_{c}$ as (Spitzer 1979)

$$
M_{\mathrm{J}}=\rho \lambda_{\mathrm{J}}^{3}=5.46\left(\frac{k T_{c}}{\mu m_{\mathrm{H}} G}\right)^{3 / 2} \rho_{c}^{-1 / 2}
$$

by adopting $T_{c}=10^{4} \mathrm{~K}$ and $\rho_{c}=\left\langle\rho_{4}\right\rangle$. The values of such Jean masses are plotted in the right-hand panels of Figure 11 and listed in the eighth column of Table 1. In the spherical model, the cooled gas in the L11 cloud has mass greater than the Jeans mass, i.e., $M_{4} \gtrsim M_{\mathrm{J}}$. In the 

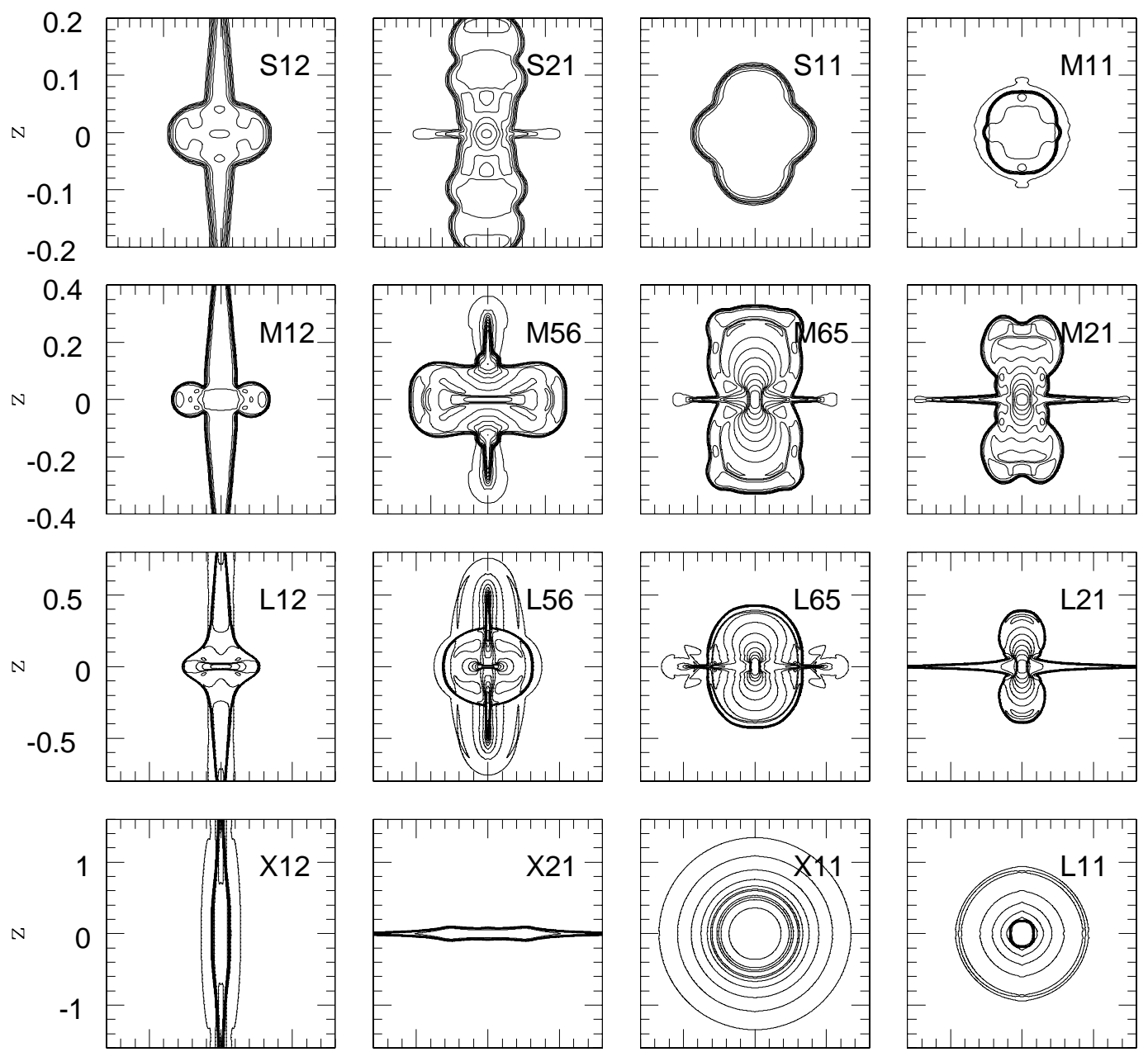

$r$

$r$

r

r

FIG. 8. - Density contour maps at $t=1.6 t_{\mathrm{cl}, h}$ for all the models with the standard cooling. The contour levels for $\left(\rho / \rho_{0}\right)=2^{i}(i=0,1, \ldots, 10)$ are shown. Each map is labeled with the model name (see Table 1).

nonspherical models, the total mass of the cooled gas in the L56, L65, and L21 clouds exceeds the Jeans mass. These clouds might become gravitationally unstable after cooling to $10^{4} \mathrm{~K}$. In those models, the clouds might imprint the Jeans mass of $M_{\mathrm{J}} \sim 5 \times 10^{7} M_{\odot}$. Assuming a star formation efficiency of order $10 \%$, this mass estimate is a bit too large for the characteristic mass scale of the GC mass distribution. This mass scale, however, is much larger than the previous estimation by Kang et al. (2000). This is because the cloud density increases only to $\rho_{c} \approx 100 \rho_{h}$ in the current simulations where the slower nonequilibrium cooling rate has been adopted. In any case, our estimation for the gravitationally unstable mass scale should be taken to be very rough and would depend on the model parameters, including the density and temperature of the background. In addition, self-gravity has been ignored in our simulations, which is expected to be important in the later stage of the cloud evolution, as pointed out in $\S 1$. Because the X11, X12, and X21 models have either small density enhancement or extremely nonspherical mass distribution, we argue that it would not be useful to make the comparison between $M_{4}$ and $M_{\mathrm{J}}$ for those models.

\subsection{Different Cooling Model}

Finally, we have calculated the L56 and L65 models with different coolings, to see their effects. They are labeled as EL56 and EL65 for the models with the CIEm0 cooling and as ML56 and ML65 for the models with the NEQm1 cooling (see also Table 1). Figure 12 shows the density contour maps at the shock formation time $\left(t=0.8 t_{\mathrm{cl}, h}\right)$ and at a later time $\left(t=1.4 t_{\mathrm{cl}, h}\right)$. In the ML56 and ML65 models where cooling is enhanced by about a factor of 3.5 at $T \sim 10^{6} \mathrm{~K}$ as a result of metals, the cloud gas has cooled to $T=10^{4} \mathrm{~K}$ already at $t=0.2 t_{\mathrm{cl}, h}$ and the compression wave steepens into a pair of shocks (reverse and forward) by $t=0.8 t_{\mathrm{cl}, h}$. This produces an elongated ringlike structure in the twodimensional image, which is in fact a dense elongated shell in three dimensions. This structure, along with the pair of shocks, collapses at the center, and then an accretion shock bounces back before $t=t_{\mathrm{cl}, h}$. The gas of the EL56 and EL65 models with the CIEm0 cooling cools faster, and so we see that the resulting structure is more compact than that in the models with the standard NEQm0 cooling. As a result, the density enhancement reaches $\left\langle\rho_{4}\right\rangle=(360-860) \rho_{h}$ by 

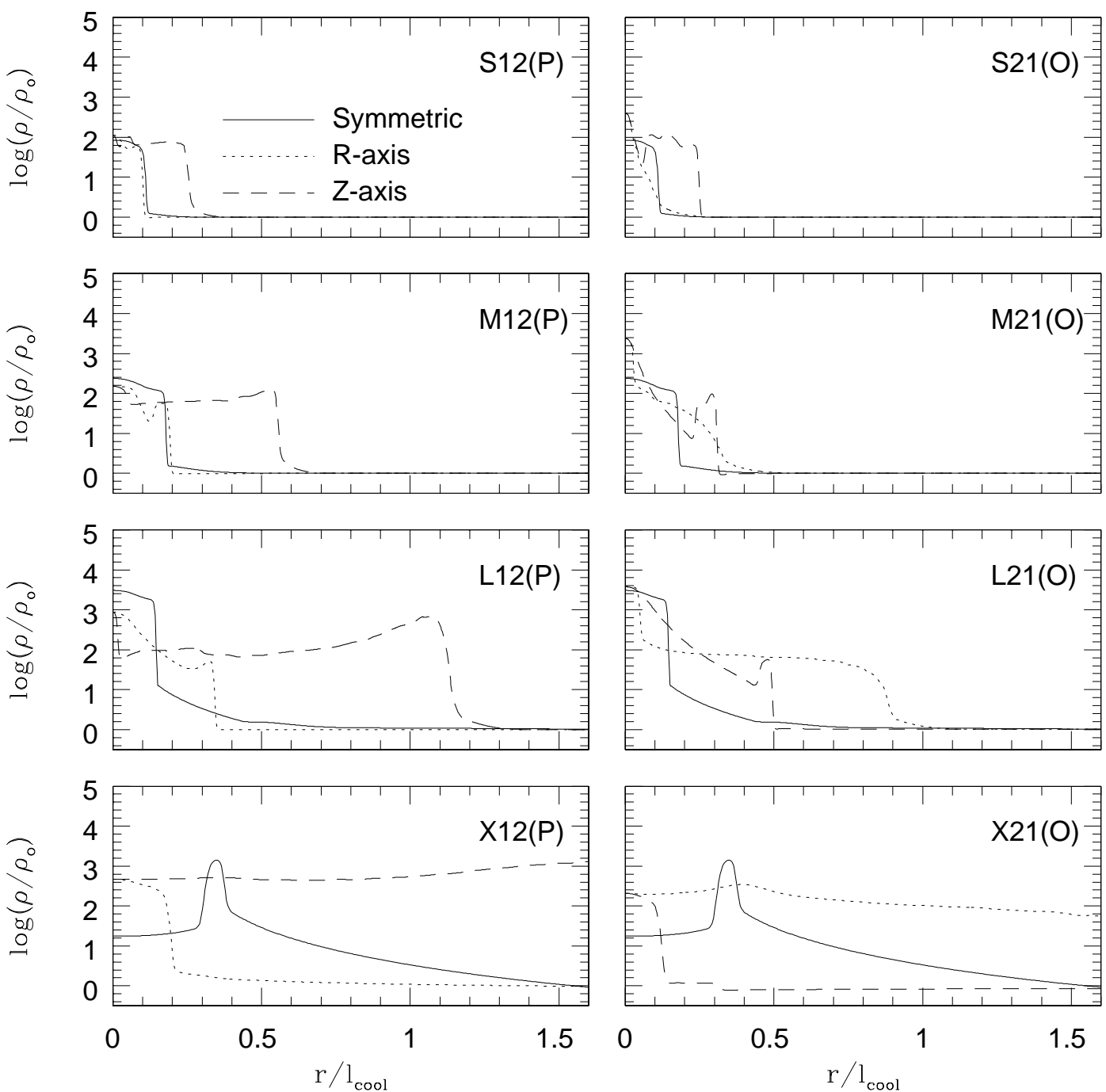

Fig. 9.-Density line profiles of the cooled clouds for the S12 (top left), S21 (top right), M12 (upper middle left), and M21 (upper middle right) models at $t=1.2 t_{\mathrm{cl}, h}$ and for the L12 (lower middle left), L21 (lower middle right), X12 (bottom left), and X21 (bottom right) models at $t=1.4 t_{\mathrm{cl}, h}$. The dotted and dashed lines show the profiles along the $R$ - and $z$-axes, respectively. The results of one-dimensional spherical simulations (the S11, M11, L11, and X11 models) are shown with the solid lines for comparison.

$t=1.4 t_{\mathrm{cl}, h}$, which are greater than those found in the models with the standard cooling. Thus, the spherical Jeans mass of the cooled clouds becomes $\sim 10^{7} M_{\odot}$, which is more consistent with the characteristic mass scale of GCs (see Table 1).

\section{SUMMARY}

In many astrophysical problems, a two-phase medium may develop by the formation of cold dense clouds via the thermal instability. In order to explore how the nonspherical shape affects the collapse of thermally unstable clouds, we have performed two-dimensional hydrodynamical simulations in the cylindrical geometry with the radiative cooling rate for a primordial gas. Although we have selected for our simulations a physical environment relevant to a protogalactic halo, the overall simulation results can be applied to the collapse of thermally unstable clouds of any physical scale, as long as the cooling curve has the pertinent characteristics for the thermal instability.

The collapse of nonspherical clouds depends mainly on the following factors:
1. The ratio of the cloud size to the cooling length, $R_{c} / l_{\text {cool }}$. As shown in the previous one-dimensional spherical simulations (e.g., David et al. 1988; Brinkman et al. 1990; Kang et al. 2000), clouds of $R_{c} \sim l_{\text {cool }}$ undergo a supersonic compression, resulting in high-density enhancements. Small clouds with $R_{c}<l_{\text {cool }}$ cool nearly isobarically through a quasi-static compression, while large clouds with $R_{c}>l_{\text {cool }}$ cool nearly isochorically. Hence, we have focused on the clouds with $R_{c} \sim l_{\text {cool }}$.

2. The initial density contrast between the cloud and the background, $(1+\delta)$. Note that the contrast in cooling times, $t_{\mathrm{cl}, c} / t_{\mathrm{cl}, h}$, is initially proportional to $(1+\delta)^{-2}$ for an isobaric perturbation. Since we have not included any heating source that maintains the temperature of the background gas, the growth of nonlinear structures depends on the value of $\delta$. For example, with $\delta \ll 1$, only the first stage of nonlinear growth can develop before the background gas itself cools down. With $\delta \sim 1$, however, more complex structures form after the initial emergence of the shape instabilities. Thus, we have started our simulations with $\delta=1$. 

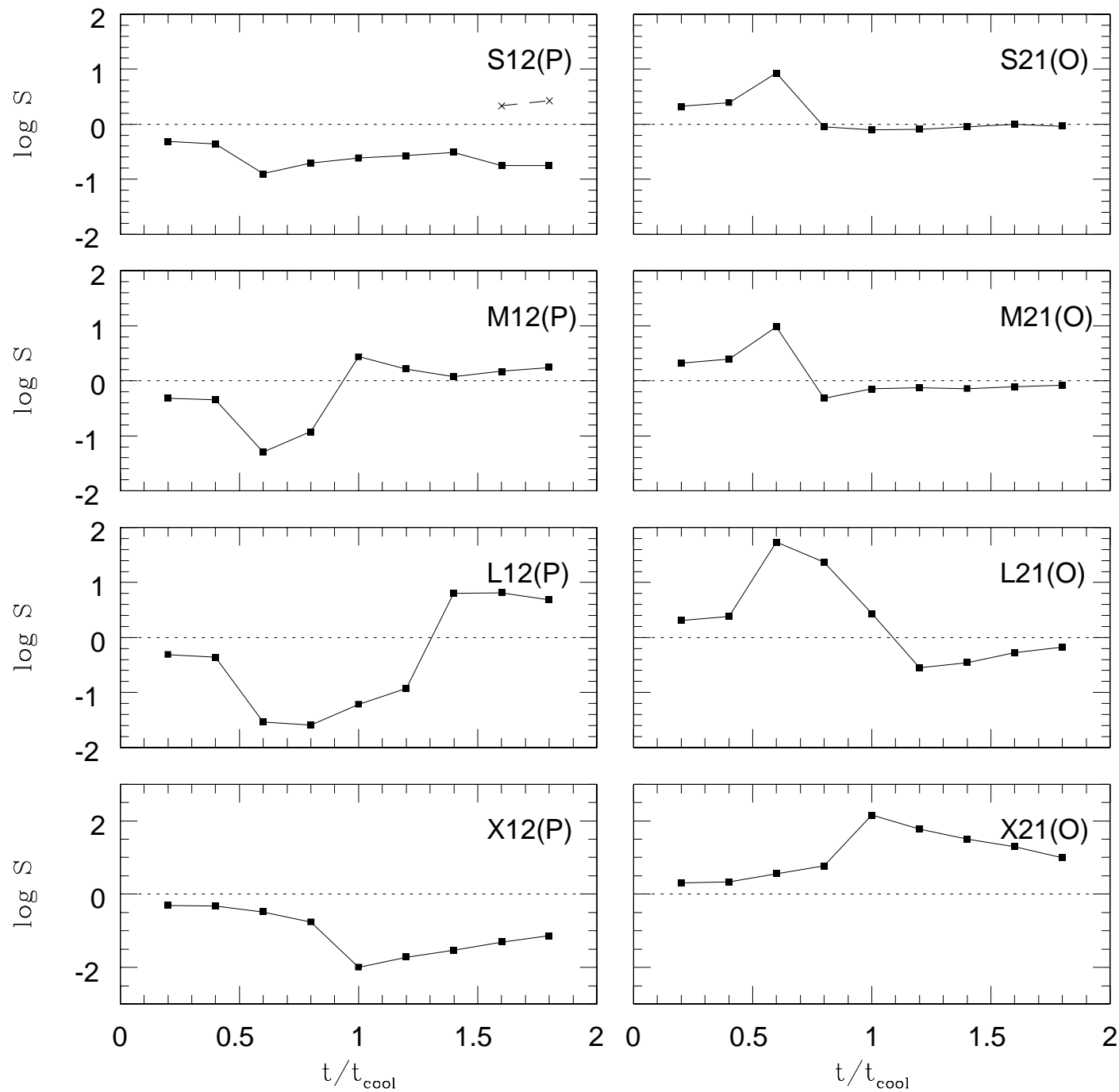

FIG. 10.- Time evolution of the shape parameter, $S$, for the prolate (left-hand panels) and oblate (right-hand panels) clouds. The dotted horizontal lines are applied to the spherical symmetry $(S=1)$. In the $\mathrm{S} 12$ model, the second shape parameter, $S_{100}$, is plotted for $t \geq 1.6 t_{\mathrm{cl}, h}$ with a dashed line.

3. The degree of nonsphericity, $S=R_{c, R} / R_{c, z}$. It is obvious that the degree of deviation from the spherical symmetry determines the dynamics of the infall flows and the mass distribution of the collapsed clouds. Hence, we have considered both the prolate clouds with $S_{i}=\frac{1}{2}$ and $\frac{5}{6}$ and the oblate clouds with $S_{i}=6 / 5$ and 2 , in addition to the spherical clouds $\left(S_{i}=1\right)$.

The collapse in our simulations can be described by two distinct stages: the first shape instability stage, during which the nonsphericity grows as a result of the initial infall, and the secondary contraction stage, during which the infall occurs predominantly along the direction perpendicular to the initial infall flows and a shape reversal occurs. Even with initially only $20 \%$ deviation from the spherical shape (i.e., $S_{i}=\frac{5}{6}$ or $6 / 5$ ), a strong shape instability occurs, so the prolate clouds are compressed to thin rods and the oblate clouds are compressed to flat pancakes as a result of strongly anisotropic infall flows. The degree of prolateness or oblateness, however, is enhanced only during the initial shape instability phase up to the formation of accretion shocks. Afterward, secondary infall motions are induced, dominantly along the $z$-axis for the prolate clouds and along the $z=0$ plane for the oblate clouds. This secondary contraction parallel or perpendicular to the $z$-axis induces, within the cooled clouds, the radial outflows in the prolate models or the bipolar outflows in the oblate models, resulting in the inner central bulges with the mass distribution opposite to the initial shape. As a result, initially prolate clouds collapse to a system that consists of an outer cigarshaped component and a central oblate component, while initially oblate clouds collapse to a system that consists of an outer pancake-shaped component and a central prolate component.

The central density of the collapsed clouds increases until accretion shocks form at the end of the first shape instability stage. Then, it gradually decreases as the clouds expand, since the central pressure is higher than the background pressure. The central density in our simulations has turned out to be much lower than that in the previous simulations. In the secondary contraction stage, the mean density of the outer pancake or thin rod components is similar to the background density times the isobaric compression ratio of $\left(T_{h} \mu_{c} / T_{c} \mu_{h}\right)=10^{2.5}$, 

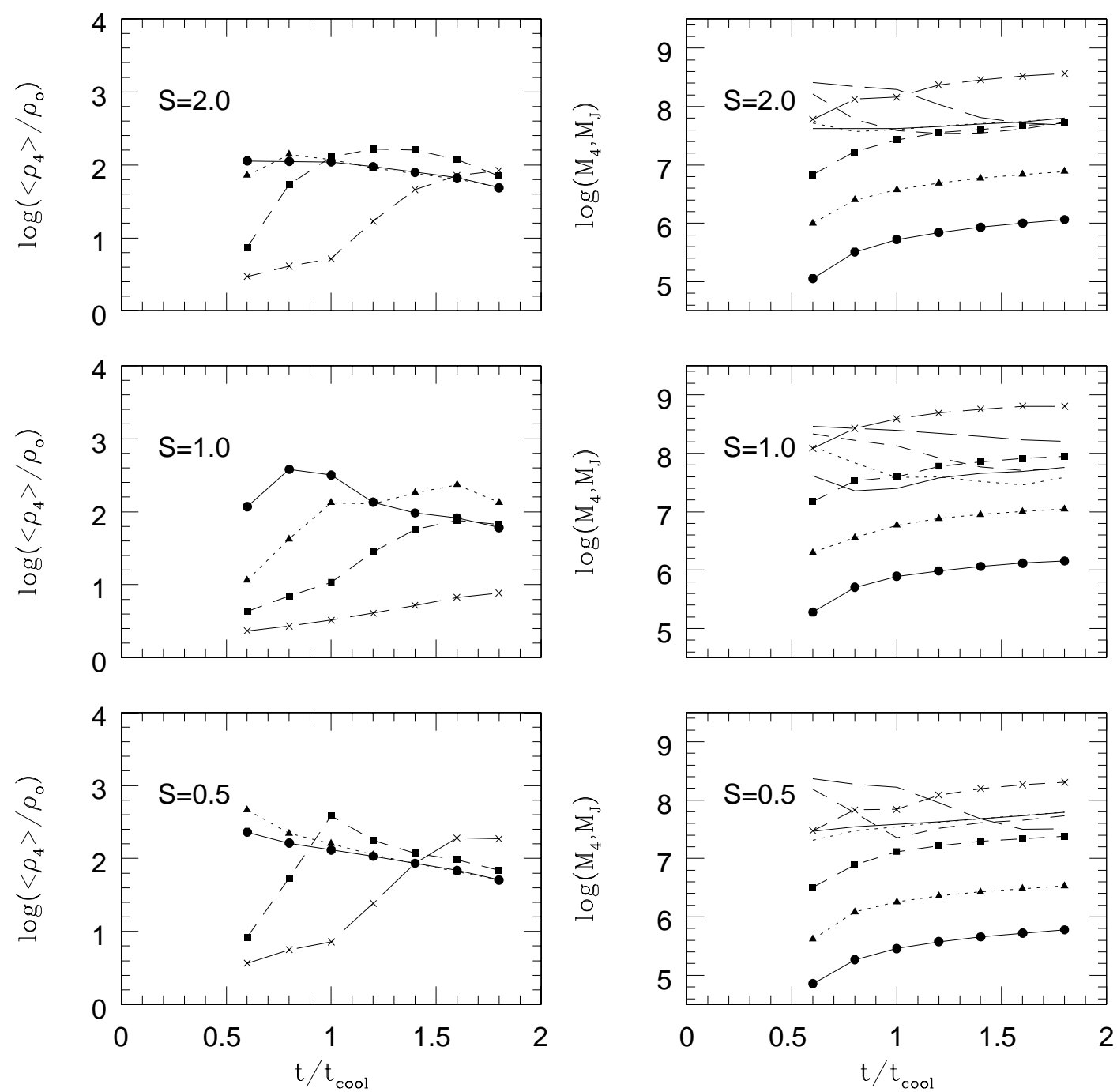

FIG. 11.-Left-hand panels: Mean density of the gas with $T=10^{4} \mathrm{~K},\left\langle\rho_{4}\right\rangle$, for prolate, spherical, and oblate clouds (from bottom to top) shown as a function of time. The solid lines with circles are for the small cloud models, the dotted lines with triangles for the medium-size cloud models, the dashed lines with squares for the large cloud models, and the long-dashed lines with crosses for the very large cloud models. Right-hand panels: Total mass of the gas with $T=10^{4} \mathrm{~K}, M_{4}$, shown with the same line types and symbols as for $\left\langle\rho_{4}\right\rangle$. The spherical Jeans mass estimated with $T_{c}=10^{4} \mathrm{~K}$ and $\left\langle\rho_{4}\right\rangle, M_{\mathrm{J}}$, is also shown in right-hand panels with the same line types but without symbols.

while that of the inner components reaches only up to an order of magnitude higher than that. We note that the density enhancement depends on the radiative cooling rate. It would be higher in the simulations with larger cooling rates. We have adopted as the standard cooling model the cooling rate of a primordial gas based on the nonequilibrium ionization fraction tabulated by Sutherland \& Dopita (1993). This cooling model has lower rates near $\mathrm{H}$ and $\mathrm{He} \mathrm{Ly} \alpha$ line emission peaks than the cooling model based on the collisional ionization equilibrium that was adopted in our previous one-dimensional simulations (Kang et al. 2000). Thus, we have found smaller density enhancements in the current simulations.

For the protogalactic halo environment considered here, $\rho_{h}=2.34 \times 10^{-25} \mathrm{~g} \mathrm{~cm}^{-3}$ and $T_{h}=1.7 \times 10^{6} \mathrm{~K}$, the spherical Jeans mass of the cooled clouds is about $M_{\mathrm{J}} \sim 5 \times 10^{7} M_{\odot}$. This mass scale is somewhat large for the mass of PGCCs that fragment to form GCs. However, this is based on a very rough estimation that would depend on model parameters. In addition, in a realistic halo environment, the halo gas may be heated by the stellar winds, supernova explosions, shock waves, etc. If the halo could maintain the high temperature and continue to compress the PGCCs, the density of PGCCs would have increased more, resulting in a smaller Jeans mass. Finally, we have considered here a static halo of uniform density. However, protogalactic halos are likely clumpy and turbulent, and the PGCCs may have formed in such an environment. We leave all these issues, along with extension into three dimensions, to future works.

This work was supported by grant R01-1999-00023 from the Korea Science and Engineering Foundation. The numerical calculations were performed through "The 2nd Supercomputing Application Support Program" of the Korea Institute of Science and Technology Information (KISTI). We thank the anonymous referee for clarifying comments. 


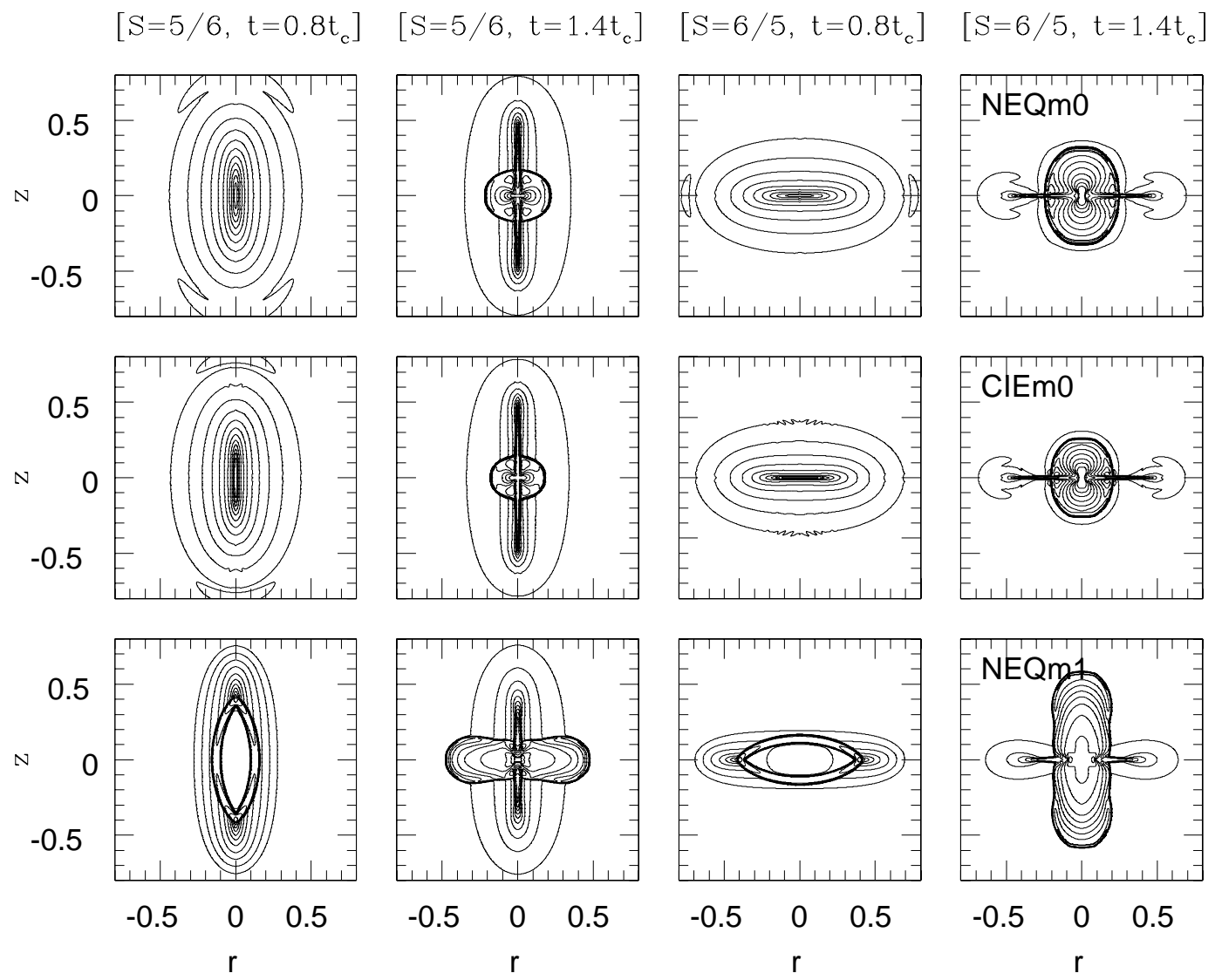

FIG. 12.-Density contour maps at $t=0.8 t_{\mathrm{cl}, h}$ and $1.4 t_{\mathrm{cl}, h}$ for the large cloud models (L56 and L65) with three different cooling models, NEQm0, CIEm0, and NEQm1 (from top to bottom).

Balbus, S. A., \& Soker, N. 1989, ApJ, 341, 611

Brinkman, W., Massaglia, S., \& Müller, E. 1990, A\&A, 237, 536

Brown, J. H., Burkert, A., \& Truran, J. W. 1991, ApJ, 376, 115

David, L. P., Bregman, J. N., \& Seab, C. G. 1988, ApJ, 329, 66

Defouw, R. J. 1970, ApJ, 160, 659

Fall, S. M., \& Rees, J. M. 1985, ApJ, 298, 18

Field, G. B. 1965, ApJ, 142, 531

Goldsmith, D. W. 1970, ApJ, 161, 41

Gray, D. R., \& Kilkenny, J. D. 1980, Plasma Phys., 22, 81

Gunn, J. E. 1980, in Globular Clusters, ed D. Hanes \& B. Madore (New

York: Cambridge Univ. Press), 301

Kang, H., Lake, G., \& Ryu, D. 2000, J. Korean Astrophys. Soc., 33, 111

Kang, H., Shapiro, P. R., Fall, S. M., \& Rees, J. M. 1990, ApJ, 363, 488

Koyama, K., \& Inutsuka, S. 2002, ApJ, 564, L97

\section{REFERENCES}

Kritsuk, A. G., \& Norman, M. L. 2002, ApJ, 569, L127

Kumai, Y., Basu, B., \& Fujimoto, M. 1993, ApJ, 404, 144

LeVeque, R. J. 1997, Computational Methods in Astrophysical Fluid Flows (27th Saas-Fee Advanced Course Lecture Notes; Berlin: Springer)

McCrea, W. H. 1957, MNRAS, 117, 562

Ryu, D., Ostriker, J. P., Kang, H., \& Cen, R. 1993, ApJ, 414, 1

Schwarz, J., McCray, R., \& Stein, R. F. 1972, ApJ, 175, 673

Shapiro, P. R., \& Kang, H. 1987, ApJ, 318, 32

Spitzer, L., Jr. 1979, Physical Processes in the Interstellar Medium (New York: Wiley)

Strang, G. 1968, SIAM J. Numer. Anal., 5, 505

Sutherland, R. S., \& Dopita, M. A. 1993, ApJS, 88, 253

Vázquez-Semadeni, E., Gazol, A., \& Scalo, J. 2000, ApJ, 540, 271 